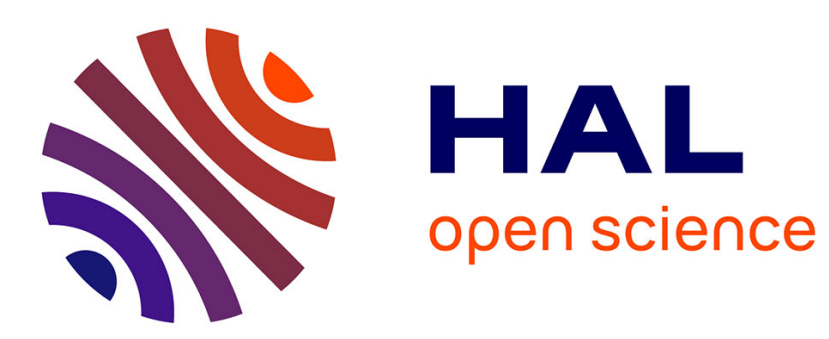

\title{
Numerical modeling of pile penetration in silica sands considering the effect of grain breakage
}

Yin-Fu Jin, Zhen-Yu Yin, Ze-Xiang Wu, Ali Daouadji

\section{To cite this version:}

Yin-Fu Jin, Zhen-Yu Yin, Ze-Xiang Wu, Ali Daouadji. Numerical modeling of pile penetration in silica sands considering the effect of grain breakage. Finite Elements in Analysis and Design, 2018, 144, pp.15-29. 10.1016/j.finel.2018.02.003 . hal-01727495

\section{HAL Id: hal-01727495 \\ https://hal.science/hal-01727495}

Submitted on 22 Oct 2019

HAL is a multi-disciplinary open access archive for the deposit and dissemination of scientific research documents, whether they are published or not. The documents may come from teaching and research institutions in France or abroad, or from public or private research centers.
L'archive ouverte pluridisciplinaire HAL, est destinée au dépôt et à la diffusion de documents scientifiques de niveau recherche, publiés ou non, émanant des établissements d'enseignement et de recherche français ou étrangers, des laboratoires publics ou privés. 


\title{
Numerical modeling of pile penetration in silica sands considering the effect of grain breakage
}

\author{
Yin-Fu Jin ${ }^{\text {a,b }}$, Zhen-Yu Yin ${ }^{\text {a,b, }}$, Ze-Xiang Wu ${ }^{\text {a,b }}$, Ali Daouadji ${ }^{c}$ \\ ${ }^{a}$ Department of Civil and Environmental Engineering, The Hong Kong Polytechnic University, Hung Hom, Kowloon, Hong Kong \\ ${ }^{\mathrm{b}}$ Research Institute of Civil Engineering and Mechanics (GeM), UMR CNRS 6183, Ecole Centrale de Nantes, Nantes, 44321, France \\ ${ }^{\mathrm{c}}$ University of Lyon, INSA Lyon, Laboratoire GEOMAS, Villeurbanne 69621, France
}

Current numerical platforms rarely consider the effect of grain breakage in the design of sandy soil foundations. This paper presents an enhanced platform for large deformation analyses which considers the effect of grain breakage during pile penetration in silica sand. For this purpose, a model based on critical state theory has been developed within the framework of multisurface plasticity to account in the same constitutive platform the effect of stress dilatancy and particle fragmentation. Furthermore, to implement the underlying constitutive equations into a finite element code, a stress integration scheme has been adopted by extending a cutting plane algorithm to the model with multiple yielding mechanisms. A laboratory model test and a series of centrifuge tests of pile penetration are simulated to verify the performance of the selected constitutive approach in terms of pile resistance and grain breakage distribution, with the parameters of sand calibrated through a set of drained triaxial compression tests from low to very high confining pressure. Some extra features of the enhanced platform are also discussed, such as: i) the effect of sand crushability on pile resistance and ii) the nonlinear relation of pile resistance to sand density. The proposed findings demonstrate the capability of this numerical platform to proper design of pile foundation in sandy soils and highlight the interplay between stress dilatancy and grain breakage mechanisms during pile penetration processes.

\section{Introduction}

Pile penetration is a common geotechnical engineering problem having to do with the improvement of a foundation, and it plays an important role in non-excavation construction. Besides of the conventional design methods employing the model test or in-situ test, numerical methods are more and more adopted in order to reduce the economic and time costs. Among numerous approaches, the finite element method (FEM) is considered a beneficial tool in engineering design (e.g., Kouretzis et al. [1], Sheng et al. [2], Zhang et al. [3,4], Shen and Xu [5]; Wu et al. [6]), when compared with meshfree methods [7], discrete element method (DEM) $[8,9]$. Accordingly, a numerical platform based on FEM adopting an appropriate constitutive model would be helpful for analyzing the pile penetration considering large deformation and further estimating the pile resistance.

During the process of pile penetration, sand along the pile always undergoes a high level of stress caused by the squeezing effect, causing lateral deformation (e.g., Shen et al. [10,11]). This high level of stress can result in grain breakage, even for silica sand. The importance of this grain breakage in silica sand during pile penetration has been highlighted from a practical standpoint $[3,4,12-18]$. Numerous studies have shown that the pile resistance in crushable sand is overestimated in comparison with that expected by a conventional simulation platform without considering grain breakage [1,3,19-21]. Accordingly, the degrading effect of grain breakage on pile resistance should be considered for practical design, which poses a requirement that the constitutive model accounting for grain breakage should be employed in the numerical platform.

In past decades, pile penetration in sand has been simulated by employing different constitutive models, such as Drucker-Prager [22] and Mohr-Coulomb [22], critical-based [1], and hyperplastic [23,24] models. However, these simulations did not consider the effect of grain breakage on pile resistance, resulting in an inaccurate prediction of pile resistance. More recently, the simulations performed by Zhang et al. [3,4, 19] adopted a simple breakage model. However, the nonlinear elasticity, dilatancy, and contraction with the soil density effect were not properly considered in this model, and its parameters were not fully calibrated

\footnotetext{
* Corresponding author. Department of Civil and Environmental Engineering, The Hong Kong Polytechnic University, Hung Hom, Kowloon, Hong Kong.

E-mail address: zhenyu.yin@gmail.com (Z.-Y. Yin).
} 
against laboratory tests on the same silica sand before the simulation [3, 19].

Therefore, this paper aims to develop an enhanced numerical platform for large deformation analysis by considering the effect of grain breakage, nonlinear elasticity, and stress dilatancy with the density effect of sand during the pile penetration process. To achieve this purpose, a critical-state-based sand model within the framework of multi-surface plasticity accounting for the effects of stress dilatancy and particle crushing is first developed. Then, for the finite element implementation, the conventional cutting plane algorithm is extended for the model with multiple yielding mechanisms. Next, the proposed platform is verified by simulating a laboratory model test and a series of centrifuge tests of pile penetration in Fontainebleau sand, in which the model parameters are calibrated from various drained triaxial tests under low to high confining pressures. Finally, some extra features of the enhanced platform are discussed, such as the effect of sand crushability on pile resistance and the nonlinear relation between pile resistance and sand density.

\section{Constitutive equations: multisurface plasticity for particle breakage}

The double-yield-surface model originally developed by Hu et al. [25] and Yin et al. [26] is adopted to enhance the numerical platform for pile penetration analysis. Consistently with the elastoplastic theory, the total strain rate is composed of the elastic and plastic strain rates:

$\dot{\varepsilon}_{i j}=\dot{\varepsilon}_{i j}^{e l}+\dot{\varepsilon}_{i j}^{p l}$

where $\dot{\varepsilon}_{i j}$ denotes the $(i, j)$ the total strain rate tensor, and the superscripts $e l$ and $p l$ represent the elastic and plastic components, respectively.

The nonlinear elastic behavior is assumed to be isotropic with the bulk modulus $K$ characterized by the same expression of the shear modulus $G$, both defined by Richart et al. [27]:

$\dot{\varepsilon}_{l j}^{e l}=\frac{1+v}{E} \dot{\sigma}_{i j}^{\prime}-\dot{\sigma}_{k k}^{\prime} \delta_{i j}$

where $v$ and $E$ are Poisson's ratio and Young's modulus, $\dot{\sigma}_{i j}^{\prime}$ is the effective stress rate tensor, and $\delta_{i j}$ is the Kronecker's delta. $E$ is calculated by using the isotropic elastic bulk modulus $K$ (i.e., $E=3 K(1-2 v)$ ), which for sand is defined as:

$K=K_{0} p_{a t} \frac{(2.97-e)^{2}}{(1+e)}\left(\frac{p^{\prime}}{p_{a t}}\right)^{n}$

where $K_{0}$ and $n$ are elastic parameters, $p^{\prime}$ is the mean effective stress, and $p_{a t}$ is the atmospheric pressure $\left(p_{\text {at }}=101.3 \mathrm{kPa}\right)$.

To model in the same constitutive framework stress dilatancy and plastic compaction, the adopted model uses two yield surfaces and two potential surfaces, for shear sliding $\left(f_{S}, g_{S}\right)$ and for normal compression $(f$ ${ }_{N}, g_{N}$ ), respectively. As a result, the plastic strain rate can be expressed as:

$\dot{\varepsilon}_{i j}^{p l}=\left(\dot{\varepsilon}_{i j}^{p l}\right)_{S}+\left(\dot{\varepsilon}_{i j}^{p l}\right)_{N}=d \lambda_{S} \frac{\partial g_{S}}{\partial \sigma_{i j}^{\prime}}+d \lambda_{N} \frac{\partial g_{N}}{\partial \sigma^{\prime}{ }_{i j}}$

where the subscripts ' $S$ ' and ' $N$ ' indicate the components of the shear sliding and normal compression, respectively. The two yield surfaces in the $p^{\prime}-q$ and $e-p^{\prime}$ planes are shown in Fig. 1, which remarks the elastic region between the shear sliding surface and normal compression surface.

The yield surface for shear sliding can be expressed as,

$f_{S}=\frac{q}{p^{\prime}}-\frac{M_{p} \varepsilon_{d}^{p l}}{k_{p}+\varepsilon_{d}^{p l}}=0$

where $p^{\prime}$ is the mean effective stress, $q$ is the deviatoric stress, $k_{p}$ is related to the plastic shear modulus, $M_{\mathrm{p}}$ is the stress ratio corresponding to the

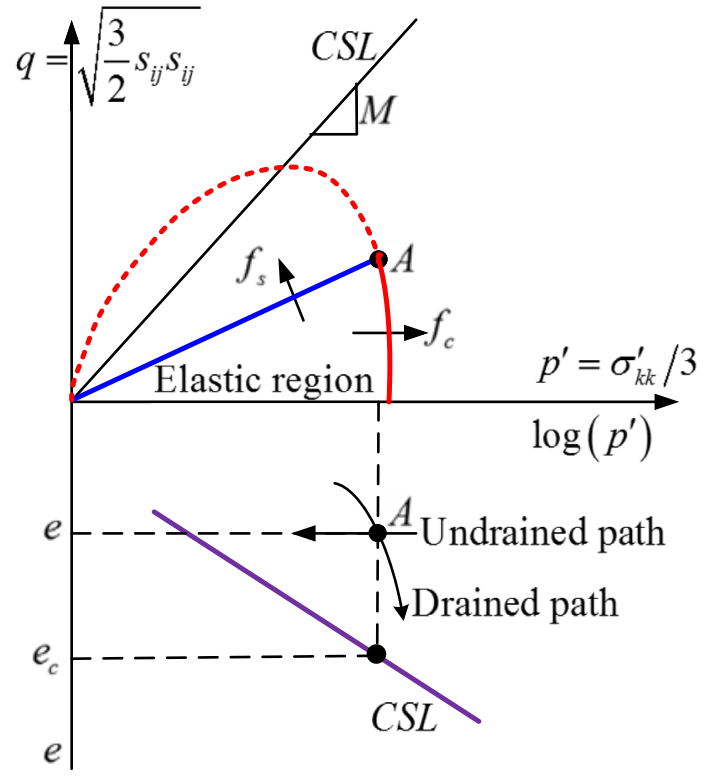

Fig. 1. Schematic representation of critical state based double-yield-surface model for crushable sand.

peak strength calculated by using the peak friction angle $\phi_{\mathrm{p}}$ (i.e., $M_{\mathrm{p}}=6 \sin \left(\phi_{\mathrm{p}}\right) /\left(3-\sin \left(\phi_{\mathrm{p}}\right)\right)$ in compression), and $\varepsilon_{d}^{p l}$ is the deviatoric plastic strain.

The gradient of the plastic potential surface for stress-dilatancy $g_{S}$ can be expressed as:

$\frac{\partial g_{S}}{\partial \sigma_{i j}}=\frac{\partial g_{S}}{\partial p^{\prime}} \frac{\partial p^{\prime}}{\partial \sigma_{i j}}+\frac{\partial g_{S}}{\partial q} \frac{\partial q}{\partial \sigma_{i j}}$ with $\frac{\partial g_{S}}{\partial p^{\prime}}=A_{d}\left(M_{p t}-\frac{q}{p^{\prime}}\right) ; \frac{\partial g_{S}}{\partial q}=1$

It is worth noting that the frictional/sliding part of the model is nonassociated as the functions $f_{S}$ and $g_{S}$ are different. $A_{d}$ is the stressdilatancy parameter, and $M_{\mathrm{pt}}$ can be calculated from the phase transformation friction angle $\phi_{\mathrm{pt}} \quad\left(M_{\mathrm{pt}}=6 \sin \left(\phi_{\mathrm{pt}}\right) /\left(3-\sin \left(\phi_{\mathrm{pt}}\right)\right) \quad\right.$ in compression).

The nonlinear critical state line (CSL) formulation proposed by Li and Wang [28] was well suited to sand modeling.

$e_{c}=e_{r e f}-\lambda\left(\frac{p^{\prime}}{p_{a t}}\right)^{\xi}$

where $e_{\mathrm{c}}$ is the critical void ratio, $e_{r e f}$ is the initial critical void ratio at $p^{\prime}=0$, and $\lambda$ and $\xi$ are the parameters controlling the shape of CSL in the $e$-log $p^{\prime}$ plane. However, for high levels of stress, this equation cannot guarantee the positiveness of the critical void ratio. Therefore, a modified version of this equation is employed by using a logarithmic scale for the void ratio:

$\log e_{c}=\log e_{r e f}-\lambda\left(\frac{p^{\prime}}{p_{a t}}\right)^{\xi} \Rightarrow e_{c}=e_{r e f} \exp \left[-\lambda\left(\frac{p^{\prime}}{p_{a t}}\right)^{\xi}\right]$

Soil density and interlocking grains effects are introduced through the expression of the friction angle as:

$\tan \phi_{p}=\left(\frac{e_{c}}{e}\right)^{n_{p}} \tan \phi_{u} ; \tan \phi_{p t}=\left(\frac{e_{c}}{e}\right)^{-n_{d}} \tan \phi_{\mu}$

where the parameters $n_{\mathrm{p}}$ and $n_{\mathrm{d}}$ are material constants, and $\phi_{\mu}$ is the friction angle at critical state. The Lode angle dependent strength and stress-dilatancy are introduced as described in Sheng et al. [29], but could also be incorporated by using the transformed stress method of Yao et al. [30-33].

To describe the compression behavior resulting from particle frag- 
mentation, the same function is considered for the yield surface and the plastic potential surface of the model (i.e., cap plasticity is considered as associated):

$f_{N}=g_{N}=\frac{1}{2}\left(\frac{q}{M_{p} p^{\prime}}\right)^{3} p^{\prime}+p^{\prime}-p_{c}$

where $p_{\mathrm{c}}$ is the hardening parameter controlling the size of the yield surface. The yield surface expands with the increasing of plastic volumetric strain, similar to the hardening rule of the Cam-Clay model,

$d p_{c}=p_{c} \frac{1+e}{\left(\lambda^{\prime}-\kappa^{\prime}\right) e} d \varepsilon_{v}^{p}$

where $\kappa^{\prime}=p^{\prime}(1+e) /(K)$ is back-calculated from the bulk modulus $K$ by using Eq. (3), and is defined in the double logarithmic space loge-log $p^{\prime}$. The parameter $\lambda^{\prime}$ controls the slope of the compression line under a high level of stress pursuant to the double logarithmic plane loge-log $p^{\prime}$.

The evolution of the critical state line resulting from crushing and particle fragmentation is described by the change of $e_{\text {ref, according to Liu }}$ et al. [34]:

$e_{\text {ref }}=e_{\text {refu }}+\left(e_{\text {refo } 0}-e_{\text {refu }}\right) \exp \left(-\rho B_{r}^{*}\right)$

where $e_{\text {refo }}$ and $e_{\text {refu }}$ are the virgin and ultimate initial critical void ratios corresponding to virgin soil without grain breakage and fractal crushed soil respectively. $\rho$ is the material constant which controls the decreasing rate of CSL due to grain breakage, while $B_{\mathrm{r}}{ }^{*}$ is the modified grain breakage index (see Zhang et al. [3,4]), which can be used to determine the current grain size distribution (GSD) of the crushed sand,

$F(d)=\left(1-B_{r}^{*}\right) F_{0}(d)+B_{r}^{*} F_{u}(d)$

where $F_{0}(d)$ and $F_{\mathrm{u}}(d)$ are respectively the initial and fractal GSDs, respectively, with $F_{\mathrm{u}}(d)=\left(d / d_{\mathrm{M}}\right)^{0.3}$ (d is the particle size and $d_{\mathrm{M}}$ is the work using the energy approach [36-38]:

$B_{r}^{*}=\frac{w_{p}}{b+w_{p}}$ with $w_{p}=\int\left(p^{\prime}\left\langle d \varepsilon_{v}^{p l}\right\rangle+q d \varepsilon_{d}^{p l}\right)$

where \langle\rangle are the McCauley brackets, $w_{p}$ is the plastic work, $b$ is a material constant controlling the evolution rate of the modified grain breakage index, with $\varepsilon_{v}^{p l}$ and $\varepsilon_{d}^{p l}$ indicate the increments of volumetric and deviatoric plastic strains, respectively.

All parameters of the presented breakage model can be divided into five groups: (1) elasticity parameters $\left(G_{0}, K_{0}\right.$, and $\left.n\right)$, (2) compressionrelated parameters $\left(p_{\mathrm{c}}, \lambda^{\prime}\right),(3) C S L$-related parameters $\left(e_{\text {refo }}, \lambda, \xi\right.$, and $\left.\phi_{\mu}\right),(4)$ interlocking-related parameters $\left(A_{\mathrm{d}}, k_{\mathrm{p}}, n_{\mathrm{p}}\right.$, and $\left.n_{\mathrm{d}}\right)$, and (5) grainbreakage-related parameters $\left(e_{\text {refu }}, b\right.$, and $\rho$ ). The calibration of these parameters will be presented later on by using drained triaxial tests at different confining pressures.

\section{Numerical platform}

\subsection{Stress integration algorithm}

The adopted breakage model was implemented into ABAQUS/ Explicit as a user-defined constitutive model via material subroutine VUMAT, according to the procedure proposed by Hibbitt et al. [39]. In the Explicit strategy, for a given time step $\Delta t$, the strain increment $\Delta \varepsilon$ is first solved by using a time central-difference integration method, in which, the stress increment $\Delta \sigma$ is updated through VUMAT using the computed $\Delta \varepsilon$.To integrate the stress state for a given time step $\Delta t$, the cutting plane algorithm proposed by Ortiz and Simo [40] for one single surface was adopted and extended for the case of a double-yield-surface.

The selected algorithm is semi-implicit and can guarantee accurate and efficient results even for large time steps. For implementation purposes, the yield function is linearized to derive the plastic multiplier variables $\lambda_{\mathrm{S}}$ and $\lambda_{\mathrm{N}}$ which are calculated by solving the following system of equations:

$\left\{\begin{array}{l}f_{S}\left(\sigma+d \sigma, \varepsilon_{d}^{p l}\right)+\frac{\partial f_{S}}{\partial \sigma} D\left(-d \lambda_{S} \frac{\partial g_{S}}{\partial \sigma}-d \lambda_{N} \frac{\partial g_{N}}{\partial \sigma}\right)+\frac{\partial f_{S}}{\partial \varepsilon_{d}^{p l}}\left(d \lambda_{S} \frac{\partial g_{S}}{\partial q}+d \lambda_{N} \frac{\partial g_{N}}{\partial q}\right)=0 \\ f_{N}\left(\sigma+d \sigma, p_{c}\right)+\frac{\partial f_{N}}{\partial \sigma} D\left(-d \lambda_{S} \frac{\partial g_{S}}{\partial \sigma}-d \lambda_{N} \frac{\partial g_{N}}{\partial \sigma}\right)+\frac{\partial f_{N}}{\partial p_{c}} \frac{\partial p_{c}}{\partial \varepsilon_{v}^{p l}}\left(d \lambda_{S} \frac{\partial g_{S}}{\partial p^{\prime}}+d \lambda_{N} \frac{\partial g_{N}}{\partial p^{\prime}}\right)=0\end{array}\right.$

maximum particle size) defined according to Coop et al. [35]. The evolution of grain breakage is calculated as a function of the modified plastic with the solutions expressed as:

$$
\left\{\begin{array}{l}
d \lambda_{S}=\frac{f_{S}\left(\sigma+d \sigma, \varepsilon_{d}^{p l}\right)}{\left(\frac{\partial g_{S}}{\partial \sigma} D \frac{\partial f_{S}}{\partial \sigma}-\frac{\partial f_{S}}{\partial \varepsilon_{d}^{p l}}\right)}+\left(\frac{\left.f_{N}\left(\sigma+d \sigma, p_{c}\right) \frac{\partial g_{S}}{\partial \sigma} D \frac{\partial f_{S}}{\partial \sigma}-\frac{\partial f_{S}}{\partial \varepsilon_{d}^{p l}}\right)-f_{S}\left(\sigma+d \sigma, \varepsilon_{d}^{p l}\right)\left(\frac{\partial g_{S}}{\partial \sigma} D \frac{\partial f_{N}}{\partial \sigma}+\frac{\partial g_{S}}{\partial p^{\prime}} \frac{\partial p_{c}}{\partial \varepsilon_{v}^{p l}}\right)}{\left.\left(\frac{\partial f_{N}}{\partial q} \frac{\partial f_{S}}{\partial \varepsilon_{d}^{p l}}-\frac{\partial f_{N}}{\partial \sigma} D \frac{\partial f_{S}}{\partial \sigma}\right)\left(\frac{\partial g_{S}}{\partial \sigma} D \frac{\partial f_{N}}{\partial \sigma}+\frac{\partial p_{c}}{\partial \varepsilon_{v}^{p l}}\right)+\frac{\partial g_{S}}{\partial \sigma} D \frac{\partial f_{S}}{\partial \sigma}-\frac{\partial f_{S}}{\partial \varepsilon_{d}^{p l}}\right)\left(\frac{\partial f_{N}}{\partial p^{\prime}} \frac{\partial p_{c}}{\partial \varepsilon_{v}^{p l}}+\frac{\partial f_{N}}{\partial \sigma} D \frac{\partial f_{N}}{\partial \sigma}\right)}\right) \frac{\left(\frac{\partial f_{N}}{\partial q} \frac{\partial f_{S}}{\partial \varepsilon_{d}^{p l}}-\frac{\partial f_{N}}{\partial \sigma} \frac{\partial f_{S}}{\partial \sigma} D\right)}{\left(\frac{\partial g_{S}}{\partial \sigma} \frac{\partial f_{S}}{\partial \sigma} D-\frac{\partial f_{S}}{\partial \varepsilon_{d}^{p l}}\right)} \\
d \lambda_{N}=\frac{\left.f_{N}\left(\sigma+d \sigma, p_{c}\right) \frac{\partial g_{S}}{\partial \sigma} D \frac{\partial f_{S}}{\partial \sigma}-\frac{\partial f_{S}}{\partial \varepsilon_{d}^{p l}}\right)-f_{S}\left(\sigma+d \sigma, \varepsilon_{d}^{p l}\right)\left(\frac{\partial g_{S}}{\partial \sigma} D \frac{\partial f_{N}}{\partial \sigma}+\frac{\partial p_{c}}{\partial \varepsilon_{v}^{p l}}\right)}{\left.\left(\frac{\partial f_{N}}{\partial q} \frac{\partial f_{S}}{\partial \varepsilon_{d}^{p l}}-\frac{\partial f_{N}}{\partial \sigma} D \frac{\partial f_{S}}{\partial \sigma}\right)\left(\frac{\partial g_{S}}{\partial \sigma} D \frac{\partial f_{N}}{\partial \sigma}+\frac{\partial p_{c}}{\partial \varepsilon_{v}^{p l}}\right)+\frac{\partial g_{S}}{\partial \sigma} D \frac{\partial f_{S}}{\partial \sigma}-\frac{\partial f_{S}}{\partial \varepsilon_{d}^{p l}}\right)\left(\frac{\partial f_{N}}{\partial p^{\prime}} \frac{\partial p_{c}}{\partial \varepsilon_{v}^{p l}}+\frac{\partial f_{N}}{\partial \sigma} D \frac{\partial f_{N}}{\partial \sigma}\right)}
\end{array}\right.
$$




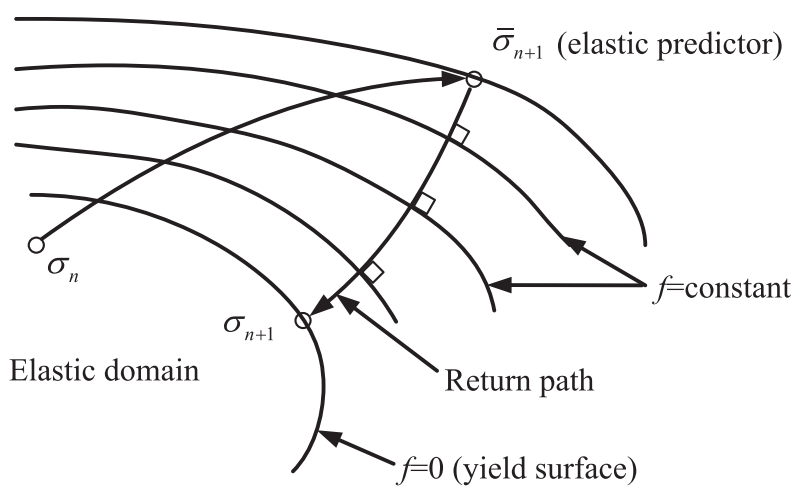

Fig. 2. Schematic diagram of a general cutting plane algorithm (after Ortiz and Simo 1986).

where $D$ is the elastic matrix.

Fig. 2 shows the schematic diagram of a general cutting plane algorithm. The purpose of the integration procedure is to compute the stress state for a given strain increment $\Delta \varepsilon$. At first, the elastic predictor $\bar{\sigma}_{n+1}$ is calculated by assuming the stress increment $\Delta \sigma$ as elastic (i.e., $\bar{\sigma}_{n+1}=\sigma_{\mathrm{n}}+\Delta \sigma=\sigma_{\mathrm{n}}+D \Delta \varepsilon$ and $\left.p_{\mathrm{cn}+1}=p_{\mathrm{cn}}\right)$ and then the corresponding value of $f=f\left(\bar{\sigma}_{n+1}\right)$ is calculated. If the value of $f$ is smaller than zero, the elastic predictor hypothesis holds and the trial stress state $\bar{\sigma}_{n+1}$ represents the solution of the integration problem for a given time step $\Delta t$. On the contrary, if $f>0$ the stress state and the hardening variable $p_{\mathrm{c}}$ has to be corrected accordingly:

$$
\left\{\begin{array}{l}
\sigma^{i+1}=\sigma^{i}-D\left(d \lambda_{S} \frac{\partial g_{S}}{\partial \sigma}+d \lambda_{N} \frac{\partial g_{N}}{\partial \sigma}\right) \\
p_{c}{ }^{i+1}=p_{c}{ }^{i}+\frac{\partial p_{c}}{\partial \varepsilon_{v}^{p l}}\left(d \lambda_{S} \frac{\partial g_{S}}{\partial p^{\prime}}+d \lambda_{N} \frac{\partial g_{N}}{\partial p^{\prime}}\right)
\end{array}\right.
$$

where the $d \lambda_{S}$ and $d \lambda_{\mathrm{N}}$ are calculated using Eq. (16). The updated values of $\sigma^{i+1}$ and $p_{c}{ }^{i+1}$ are then used to compute $f_{\mathrm{S}}$ and $f_{\mathrm{N}}$ along the return path shown in Fig. 2, until the corrected stress satisfies the plastic constraints (i.e., the convergence of the numerical algorithm is achieved when both $f$ $\mathrm{s}\left(\sigma^{i+1}, p_{c}{ }^{i+1}\right)$ and $f_{\mathrm{N}}\left(\sigma^{i+1}, p_{c}{ }^{i+1}\right)$ are less than a given tolerance, in this study equal to $10^{-7}$ ). The final stress point is obtained as $\sigma_{\mathrm{n}+1}$, which is the stress state corresponding to the strain increment $\Delta \varepsilon$. If only one yield

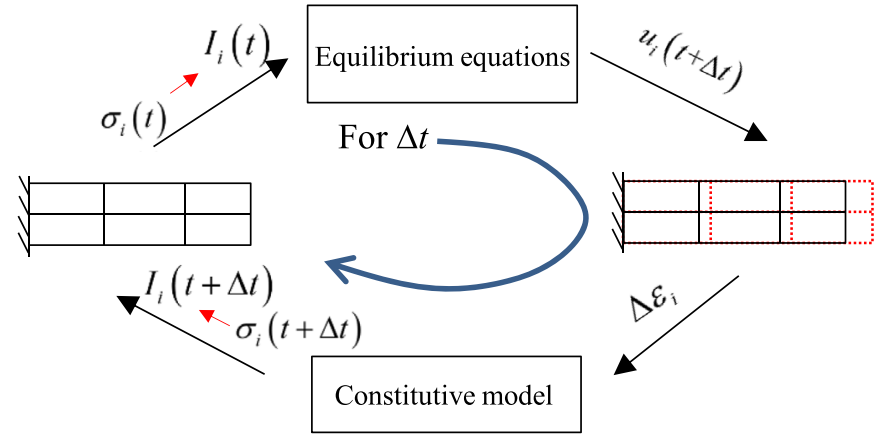

Fig. 4. Flow chart of explicit finite element analysis of ABAQUS/Explicit.

surface is activated (only $f_{S}>0$ or only $f_{N}>0$ ), the algorithm goes to the original cutting plane of Ortiz and Simo [40].

The flow-chart of this iterative strategy is schematized in Fig. 3 and all the mathematical derivations are presented in Appendix A. It is worth observing that, as the global strategy to solve the prescribed Initial Boundary Value Problem is explicit, the strain increment enforced to integrate the stress state is already very small, thus enabling the integration algorithm to reach the convergence with only few iterations.

\subsection{Explicit finite element method}

To simulate the pile penetration problem with the abovementioned constitutive framework, an explicit method implemented in ABAQUS is considered to overcome mesh distortion in problems characterized by large deformations. The underlying numerical strategy is presented in Fig. 4 with a brief introduction.

First, the equilibrium condition is written with the balance of inertial force and external force at time $t$,

$M \ddot{u}_{(t)}=P_{(t)}-I_{(t)}$

where $M$ is the mass matrix; $P$ is the applied external force vector, and $I$ is the internal force vector.

Accelerations and velocities at a particular point that result from external loads are assumed to be constant during a time increment. The equations of motion for the body are then integrated using the explicit time central-difference integration rule,

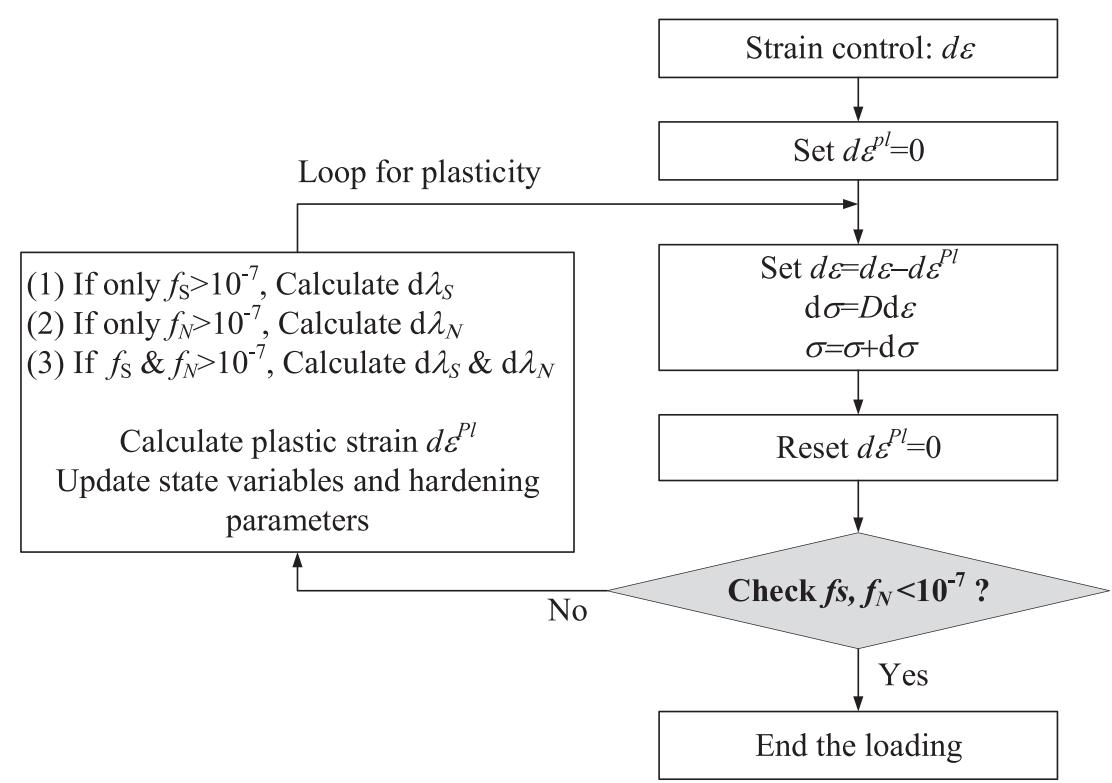

Fig. 3. Flow chart of the cutting plane algorithm. 


$$
\left\{\begin{array}{l}
\dot{u}_{\left(t+\frac{\Delta t}{2}\right)}=\dot{u}_{\left(t-\frac{\Delta t}{2}\right)}+\frac{\Delta t_{(t+\Delta t)}+\Delta t_{(t)}}{2} \ddot{u}_{(t)} \\
u_{(t+\Delta t)}=u_{(t)}+\Delta t_{(t+\Delta t)} \dot{u}_{\left(t+\frac{\Delta t}{2}\right)}
\end{array}\right.
$$

where $u$ is the displacement, the subscript $t$ refers to the time in an explicit dynamic step; $\dot{u}$ is the velocity, and $\Delta t$ is the increment of time. For the sake of numerical stability, in the explicit strategy it is recommended to prescribe a time interval $\Delta t$ smaller than a limited value which is calculated as $\Delta t \approx L_{\min } / c_{d}$, where $L_{\min }$ is the smallest element dimension of the whole mesh while $c^{\mathrm{d}}$ is expressed as a function of the Lamé coefficients $\lambda$ and $\mu$, (i.e., $c^{d}=\sqrt{(\lambda+2 \mu) / \rho}, \rho$ is material density).

The incremental displacements $\left(\Delta u=\Delta t_{(t+\Delta t)} \dot{u}_{\left(t+\frac{\Delta t}{2}\right)}\right)$ are then used to calculate the incremental strain by means of compatibility equations, which will be called by the constitutive model to update stresses and then inertial forces, up to a new equilibrium condition.

It is worth noting that, beside the purely explicit method adopted in this study, other numerical techniques such as the Coupled EulerianLagrangian method (CEL) have been successfully implemented to overcome numerical problems related to large-deformations. In particular, the performance of the CEL method to solve complex geotechnical problems have been described in Qiu et al. [24] who detail the efficiency and the robustness of this strategy with respect to other numerical methods.

\section{Model calibration for Fontainebleau sand}

The performance of the proposed constitutive platform has been studied by simulating laboratory experiments on Fontainebleau sand NE34 (i.e., tests of pile penetration and centrifuge tests) in which the material parameters have been calibrated through a series of drained triaxial tests at different soil densities and different confining pressures. The calibration procedure and the corresponding model performance are presented in the following sections.

\subsection{Determination of elasto-plastic parameters}

The parameters $K_{0}$ and $n$ were determined by fitting the isotropic compression test conducted by Gaudin et al. [41] as reported in Fig. 5(a), where for calibration purposes the $K_{0}$ and $n$ have been selected equal to 100 and 0.55 . The initial shear modulus $G_{0}$ was calculated by considering a typical value of Poisson's ratio (i.e., $v=0.25$ ) and the initial bulk modulus $K_{0}$ (i.e., $\left.G_{0}=3 K_{0}(1-2 v) / 2(1+v)=60\right)$. The yield stress corresponding to the occurrence of grain breakage $\sigma_{y}=25 \mathrm{MPa}$ and the compression coefficient $\lambda^{\prime}=0.25$ were obtained by simulating the oedometer test carried out by Yang et al. [17], shown in Fig. 5(b).

A series of drained triaxial tests performed at different confining pressures (from $50 \mathrm{kPa}$ up to $400 \mathrm{kPa}$ of confining, i.e., the tests marked as TM1 TM11 in Table 1) have been selected to calibrate the CSL and the other shear sliding parameters. These experiments are characterized by a negligible amount of grain crushing, therefore the same values of the variables $e_{\text {ref0 }}=e_{\text {refu }}$ has been considered to ignore the breakage effects in the modeling. The friction angle $\phi=33.2^{\circ}$ was measured from the $p^{\prime}-q$ plot according to the experimental results. The parameters controlling the position of CSL (i.e., $e_{r e f o}, \lambda$ and $\xi$ ), and the shear sliding (i.e., $k_{\mathrm{p}}, A_{\mathrm{d}}, n_{\mathrm{p}}$ and $n_{\mathrm{d}}$ ) can be determined by trial and error or through other optimization techniques (Wu et al., 2017 [42]). It is worth noting, that the shear test data extend only to an axial strain of $10 \%$ due to the development of shear band in the sample, therefore the critical states are not reached and cannot be measured directly. To overcome this limitation, the optimization based on inverse analysis proposed by Jin et al. [43-46] and Yin et al. $[47,48]$ was adopted in this case to identify all these model parameters using five objective tests "TM1 TM5".

By using the identified set of parameters, all tests (TM1 TM11) have been simulated at low stress levels were simulated by using an element integrated with one single Gauss point. The comparison between the numerical simulations and the experimental results are shown in Fig. 6 and Fig. 7 for the test series (TM1 TM5) and (TM6 TM11), respectively. The good agreement indicates that the model parameters identified by optimization are reliable and accurate, and the selected constitutive framework can well reproduce the state-dependent behavior of Fontainebleau sand under low confining stress with no need to consider the effects of grain breakage.

\subsection{Determination of breakage parameters}

To determine the model parameters which govern the evolution of breakage, a series of drained triaxial tests at high confining pressure has been performed on the same standard Fontainebleau sand used by Touati [49]. Table 2 shows the initial void ratios before shearing $e_{0}$, confining stresses $\sigma_{\mathrm{c}}^{\prime}$ and the final void ratio $e_{\mathrm{cf}}$ for five selected tests "TF1 TF5". For each test, the grain size distribution after shearing was also measured and used to estimate the breakage index $B_{\mathrm{r}}{ }^{*}$. The plastic work $w_{\mathrm{p}}$ was computed according to $\mathrm{Hu}$ et al. [25] and was used to determine the breakage index $B_{\mathrm{r}}{ }^{*}$ as shown in Fig. 8(a). The value $b=12000$ was obtained by fitting all the experimental data based on Eq. (14).

According to Hu et al. [25] and Yin et al. [50], the parameters $\lambda$ and $\xi$ controlling the shape of CSL can be considered the same during breakage, and only the parameter $e_{\text {ref }}$ controlling the position of CSL has been reduced. Assuming $e_{\mathrm{cf}}$ with its corresponding $p^{\prime}$ on the CSL, five $e_{\text {ref }}$ can
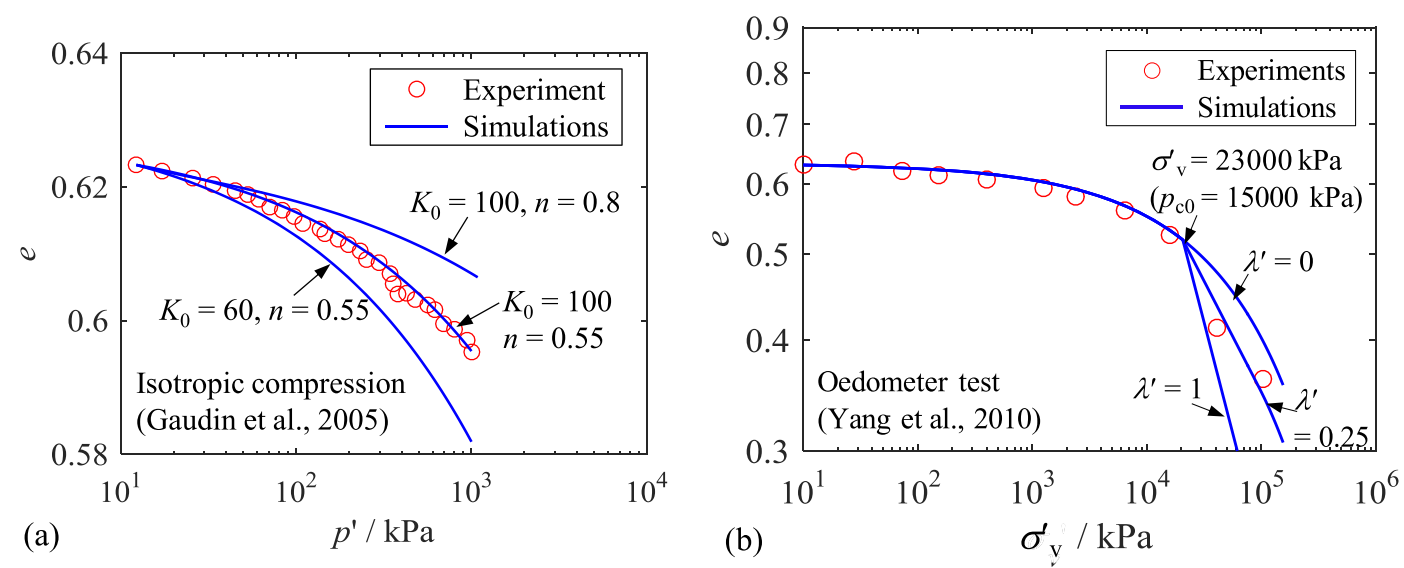

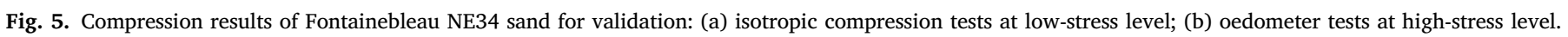


Table 1

Triaxial tests of Fontainebleau sand with different void ratios at low confining pressures.

\begin{tabular}{|c|c|c|c|c|c|c|c|c|c|c|c|}
\hline Tests & TM1 & TM2 & TM3 & TM4 & TM5 & TM6 & TM7 & TM8 & TM9 & TM10 & TM11 \\
\hline$e_{0}$ & 0.718 & 0.712 & 0.702 & 0.637 & 0.573 & 0.637 & 0.638 & 0.636 & 0.584 & 0.573 & 0.571 \\
\hline$\sigma_{\mathrm{c}}^{\prime} / \mathrm{kPa}$ & 50 & 100 & 200 & 50 & 50 & 100 & 200 & 400 & 100 & 200 & 400 \\
\hline
\end{tabular}
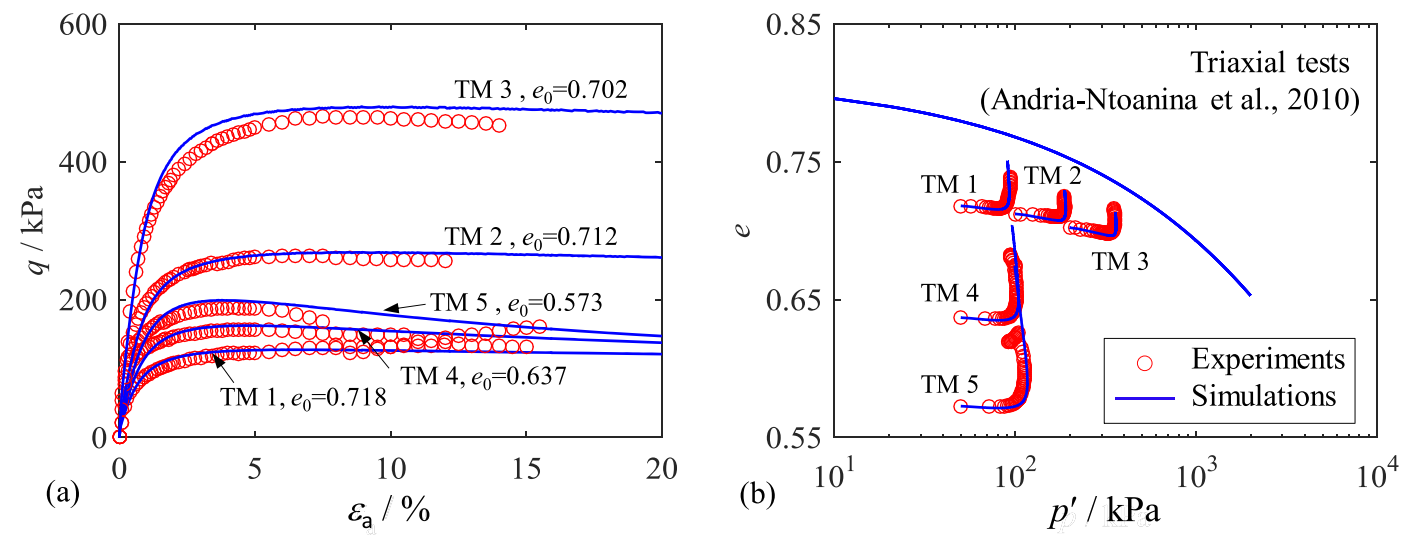

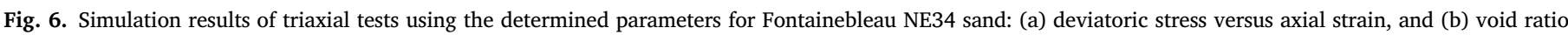
versus mean effective stress.
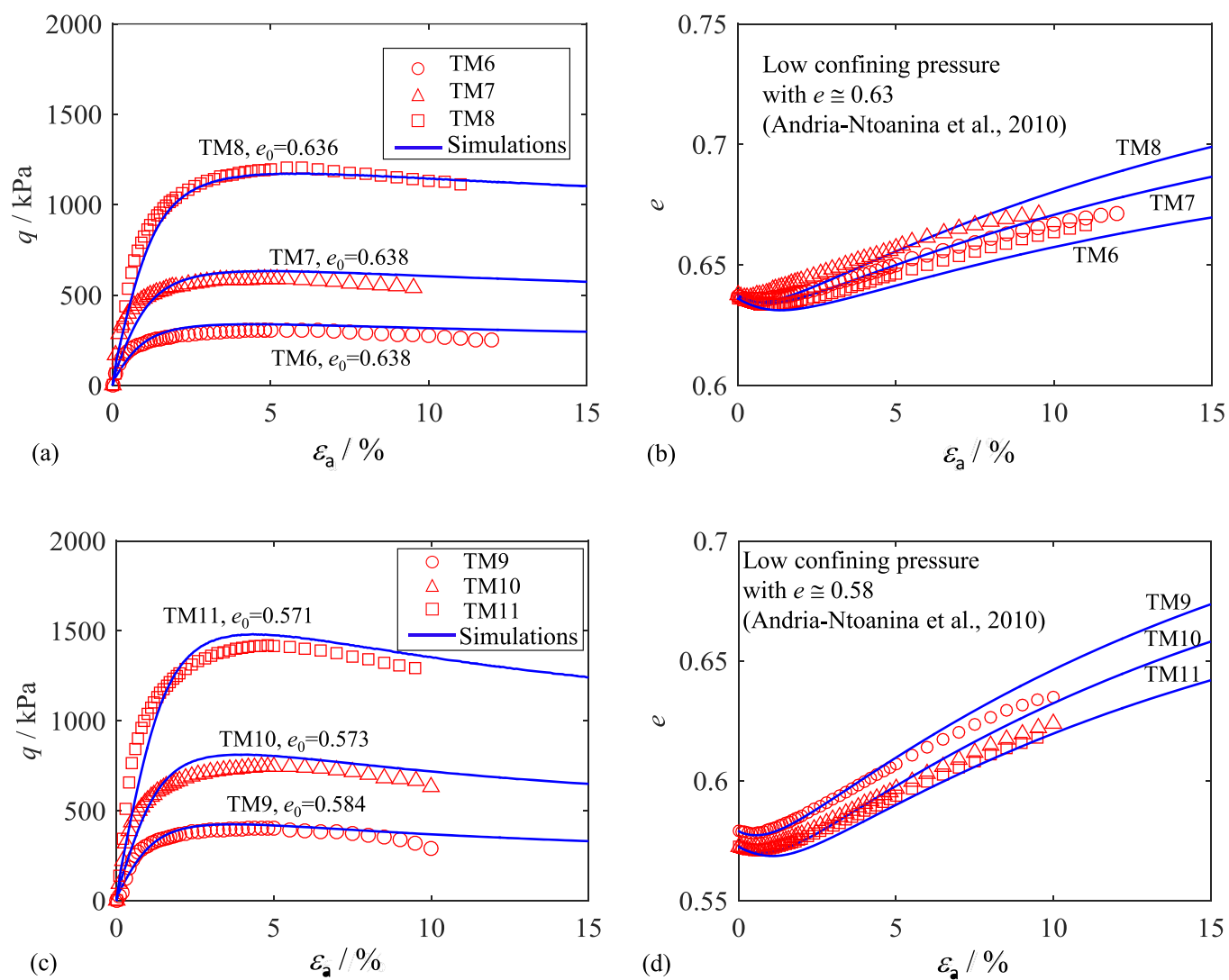

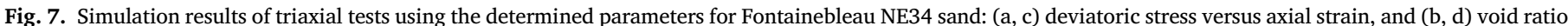
versus axial strain.

be obtained from five tests using Eq. (8). Fig. 8(b) shows the evolution of the initial critical void ratios $e_{\text {ref }}$ versus the corresponding breakage index $B_{\mathrm{r}}{ }^{*}$. Thus, $e_{\text {refu }}=0.296$ and $\rho=4$ in the Eq. (12) were obtained by fitting all measurements. All values of parameters are summarized in Table 3.

\subsection{Parameters validation}

To validate the selected set of the material parameters, the aforementioned tests "TM1 TM5" and "TF1 TF5" were set as objectives for 
Table 2

Triaxial tests under high confining pressures on Fontainebleau sand.

\begin{tabular}{llllll}
\hline Tests & TF1 & TF2 & TF3 & TF4 & TF5 \\
\hline$e_{0}$ & 0.529 & 0.522 & 0.521 & 0.502 & 0.475 \\
$\sigma^{\prime} / \mathrm{kPa}$ & 4000 & 6000 & 7000 & 16000 & 30000 \\
$e_{\mathrm{cf}}$ & 0.543 & 0.499 & 0.470 & 0.355 & 0.273 \\
\hline
\end{tabular}

the optimization. Using the same procedure as Jin et al. [43-46] (see Appendix B), all model parameters were identified and summarized in Table 3, producing results very close to the values previously determined.

To evaluate the performance of the selected parameters, the tests in Table 2 were simulated. The comparison between experiments and simulations is shown in Fig. 9 for different initial void ratios and in Fig. 10 for the grain size distribution, with good agreement for tests TF1 TF5 under high confining pressures. The low confining pressure tests were also simulated again using the final determined parameters, with almost the same simulations as previous plot (Figs. 6-7) and thus no need for repetition owing to the very high value of $b$ results for very slight changes of $B_{\mathrm{r}}{ }^{*}$ in these test simulations $\left(B_{\mathrm{r}}{ }^{*} \leq 0.02\right)$.

\section{Numerical modeling of pile penetration}

\subsection{Modeling of pile penetration in calibration chamber}

The model test of pile installation was conducted at the Institut Polytechnique de Grenoble (INPG) in the calibration chamber. This test

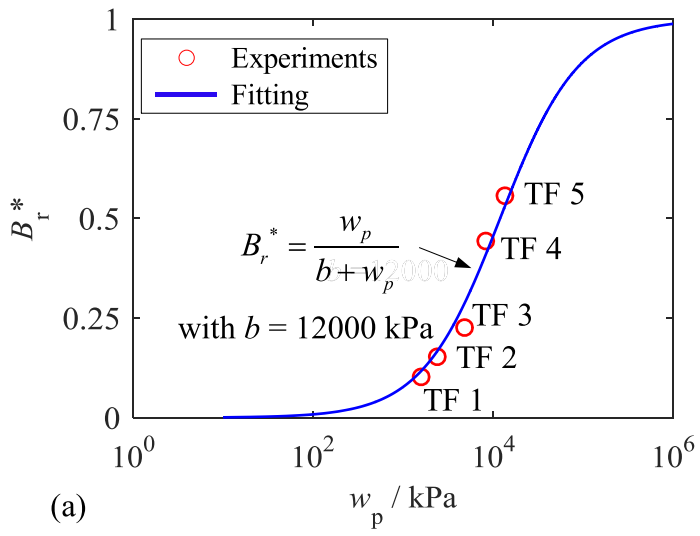

employed a Mini Imperial College Pile (Mini-ICP) which comprises an ICP type device scaled down to $36 \mathrm{~mm}$ outer diameter with a standard apex angle equal to $60^{\circ}$ closed-ended conical base, and with $1.5 \mathrm{~m}$ long for the laboratory calibration chamber testing [16]. The pile installation was displacement-controlled at $2 \mathrm{~mm} / \mathrm{s}$.

An axis-symmetric finite element model with 5700 elements was generated, as illustrated in Fig. 11. The overall model size was $0.6 \mathrm{~m}$

Table 3

Values of model parameters for Fontainebleau sand accounting for the effect of grain breakage.

\begin{tabular}{llll}
\hline Group & Parameters & Values & Optimal \\
\hline Elastic parameters & $v$ & 0.25 & 0.25 \\
& $K_{0}$ & 100 & 100 \\
CSL parameters & $n$ & 0.55 & 0.55 \\
& $e_{\text {refo }}$ & 0.825 & 0.827 \\
& $\lambda$ & 0.055 & 0.058 \\
Compression parameters & $\xi$ & 0.46 & 0.43 \\
& $\phi /^{\circ}$ & 33.2 & 33.2 \\
Shear-sliding parameters & $p_{c o} / \mathrm{kPa}$ & 15000 & 15000 \\
& $\lambda^{\prime}$ & 0.25 & 0.25 \\
& $k_{\mathrm{p}}$ & 0.0015 & 0.0017 \\
& $A_{\mathrm{d}}$ & 0.55 & 0.5 \\
Grain breakage parameters & $n_{\mathrm{p}}$ & 2.5 & 2.1 \\
& $n_{\mathrm{d}}$ & 2.8 & 3.1 \\
& $b / \mathrm{kPa}$ & 12000 & 12000 \\
& $e_{\text {refu }}$ & 0.296 & 0.296 \\
& $\rho$ & 4 & 4 \\
\hline
\end{tabular}

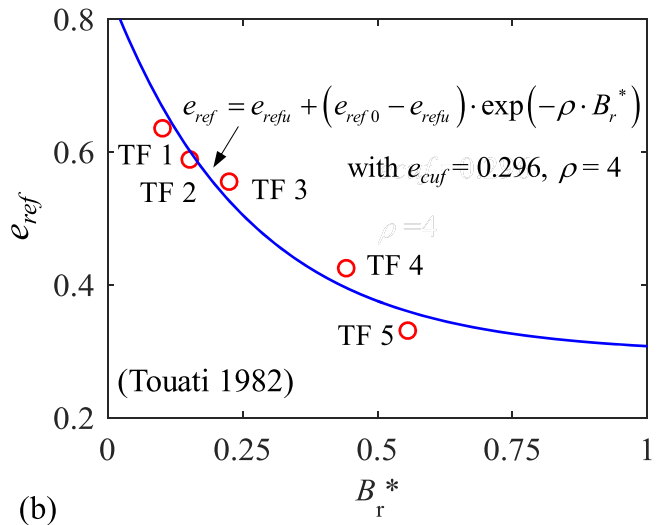

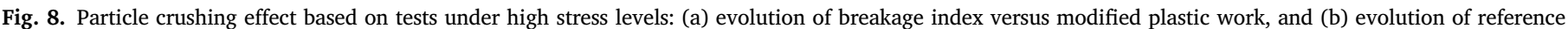
critical state void ratio versus breakage index.
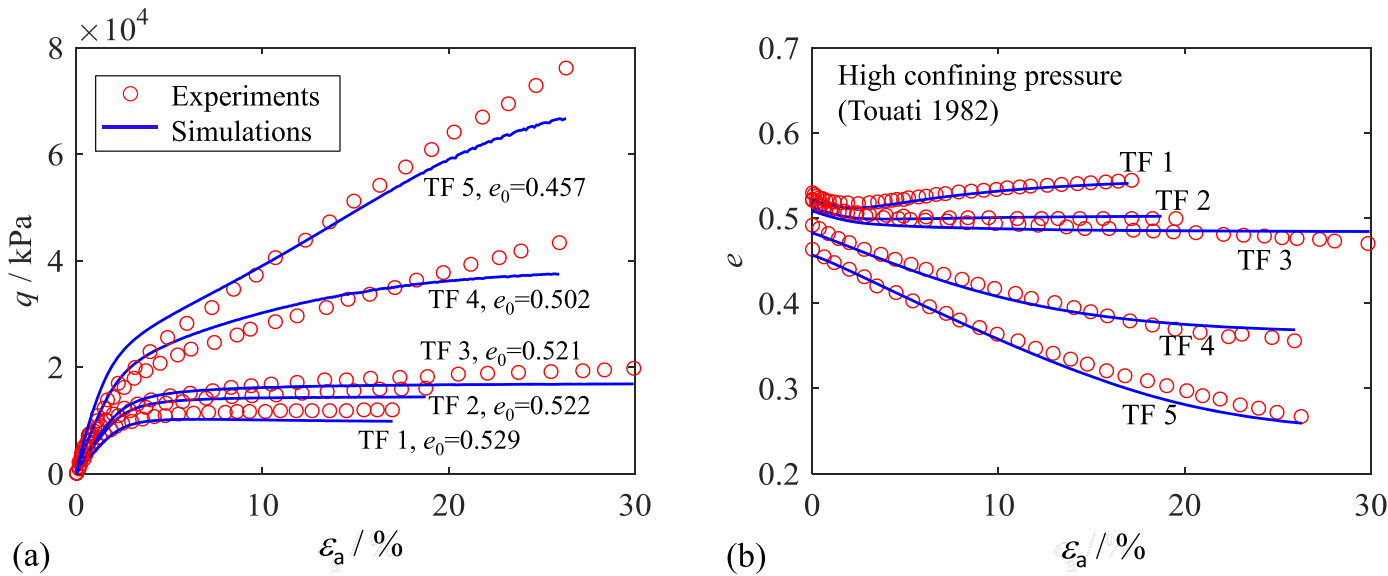

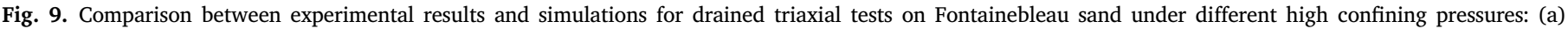
deviatoric stress versus axial strain, and (b) void ratio versus axial strain. 


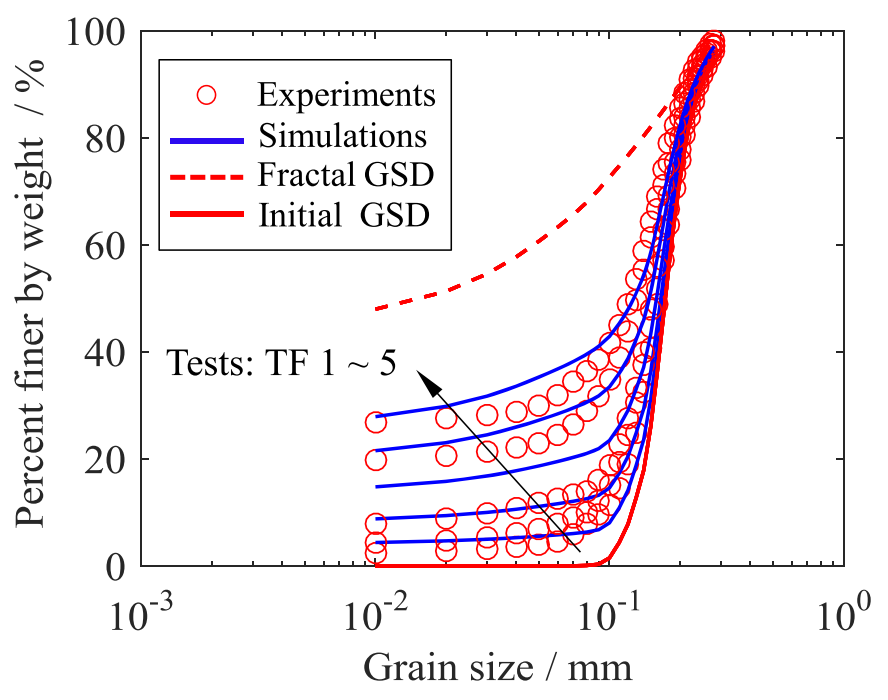

Fig. 10. Grain size distribution between experiments and simulations.
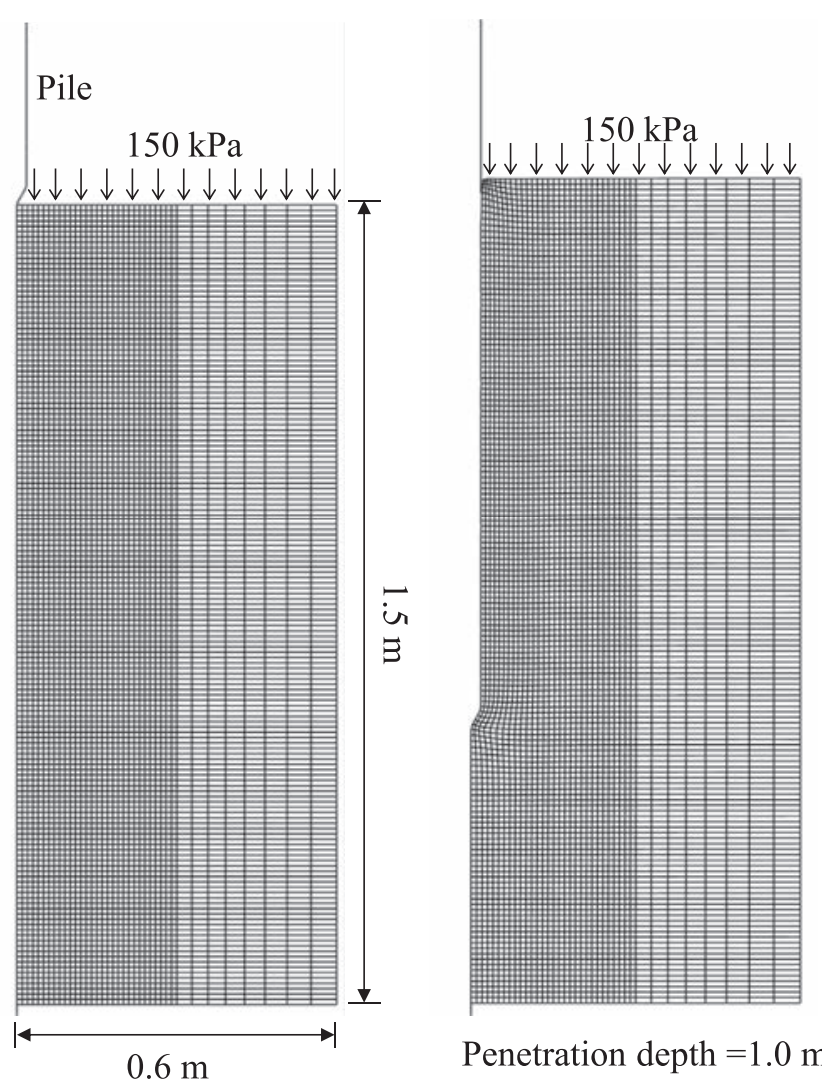

Penetration depth $=1.0 \mathrm{~m}$

Fig. 11. Geometry of ICP model pile penetration tests.

width and $1.5 \mathrm{~m}$ height, which is large enough to avoid boundary constraints. The bottom side was fixed in both vertical and horizontal directions. The left and right sides were fixed only in the horizontal direction; the top side was free. The soil was modeled by four-node axisymmetric elements with one reduced integration point (CAX4R) in ABAQUS. Compared to soil, the deformation of the pile was negligible, so the pile was modeled as a rigid body having the same diameter and conical tip as the model pile.

The initial stress was generated by self-weight with the unit weight of the soil $\gamma=16.3 \mathrm{kN} / \mathrm{m}^{3}$ under $K_{0}=1-\sin \phi_{\mu}=0.48$, which is consistent with the model test [17]. Pile-soil interaction was simulated by

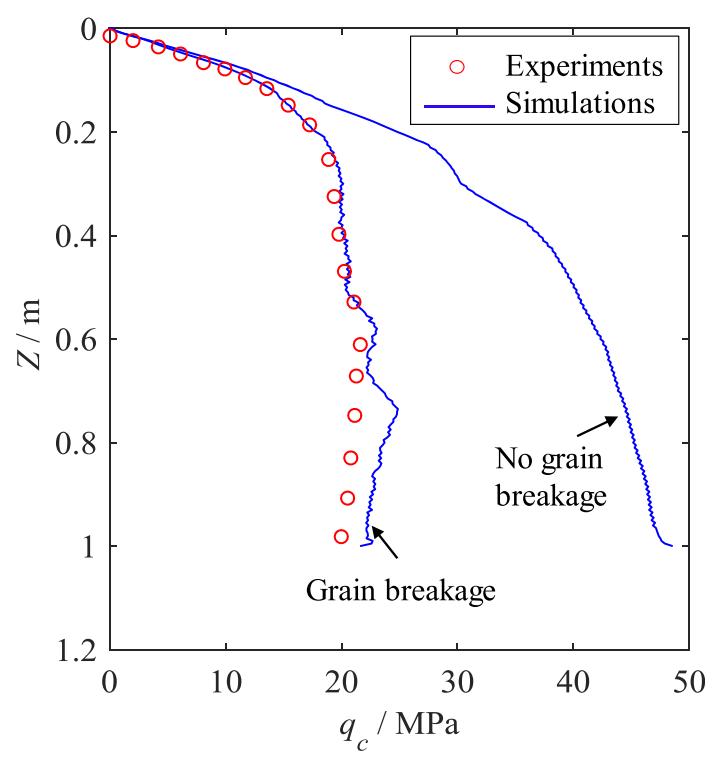

Fig. 12. Comparison between experiment and simulation of model pile test in Fontainebleau sand for pile resistance against penetration depth.

surface-to-surface contact with a classical Coulomb friction law. The friction coefficient was set to $\mu=\tan \left(\phi_{\mu} / 2\right)=0.3$. The total penetration displacement was $1000 \mathrm{~mm}$ with a slow penetration rate of $0.5 \mathrm{~mm} / \mathrm{s}$, thereby avoiding numerical issues related to the sudden application of high strain rates and inertia effects. This rate was about 4 times slower than the actual rate of the tests-an acceptable rate, according to Kouretzis et al. [1]. The parameters of Fontainebleau NE34 sand in Table 3 were used for the simulation. The simulation of pile penetration was carried out in three steps: (1) generation of initial stress, (2) application of vertical stress of $150 \mathrm{kPa}$ to the top surface of sand, and (3) penetration of the pile into sand.

In Fig. 12, the pile resistance $q_{\mathrm{c}}$ recorded during the experiments has been compared with the numerical simulation in which, to better emphasize the effect of crushing during the pile driven process, the model has been also tested without considering the effect of grain breakage (i.e., by selecting $b=0, e_{\text {refu }}=e_{\text {refo }}$, and $\rho=0$ ). It can be noticed that, consistently with the experimental observations, the breakage of sand particles during penetration dramatically decrease the pile resistance, thus confirming the good performance of the proposed constitutive platform.

Such a result is explained by considering that, the void ratio influences the mobilized soil strength, the mean effective stress influences the pile resistance, and the modified plastic work influences the amount of grain breakage, these three variables were selected to be plotted in Fig. 13(a-c). The sand is densified in the zone along the pile penetration path, especially around the pile tip, where a high-stress level is reached. Also the modified plastic work also concentrates in proximity of the pile tip, thus intensifying the sand crushing during the pile penetration.

Furthermore, to show which kind of yielding mechanism was activated at the end of the penetration, the plastic index "IPLAS" was defined as: IPLAS $=-1$ if $\left(\mathrm{d} \lambda_{\mathrm{s}}=0\right.$ and $\left.\mathrm{d} \lambda_{\mathrm{n}}=0\right)$, IPLAS $=0$ if $\left(\mathrm{d} \lambda_{\mathrm{s}}>0\right.$ and $\left.\mathrm{d} \lambda_{\mathrm{n}}=0\right)$, IPLAS $=1$ if $\left(\mathrm{d} \lambda_{\mathrm{s}}=0\right.$ and $\left.\mathrm{d} \lambda_{\mathrm{n}}>0\right)$, and IPLAS $=2$ if $\left(\mathrm{d} \lambda_{\mathrm{s}}>0\right.$ and $\left.\mathrm{d} \lambda_{\mathrm{n}}>0\right)$. The contour of this variable is plotted in Fig. 13(d) from which it is possible to observe that: (1) elements at right and upper corner remains elastic indicating no influence by the pile penetration, (2) the rest of elements exhibits plasticity only due to shearing "IPLAS $=0$ " which coincides with a stress state ( $p_{\max }=7.8 \mathrm{MPa}$ in Fig. 13(b)) lower than the compression yield stress $\left(p_{\mathrm{c} 0}=15 \mathrm{MPa}\right)$. So the grain breakage occurred in this case is totally due to the shear loading, which is consistent with many results of shear tests under low confining stresses [51-55]. 


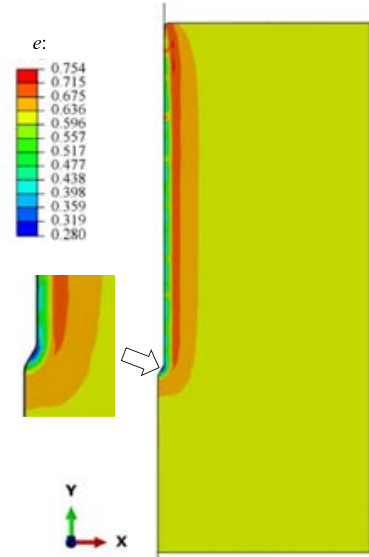

(a)

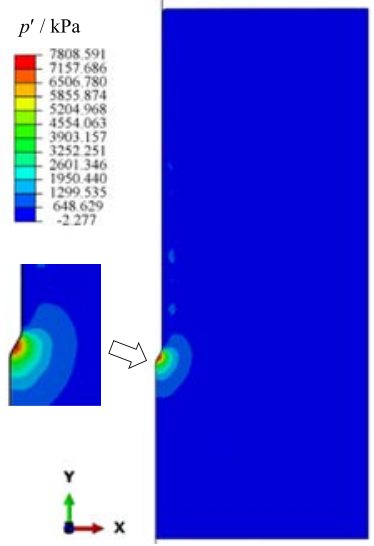

(b)

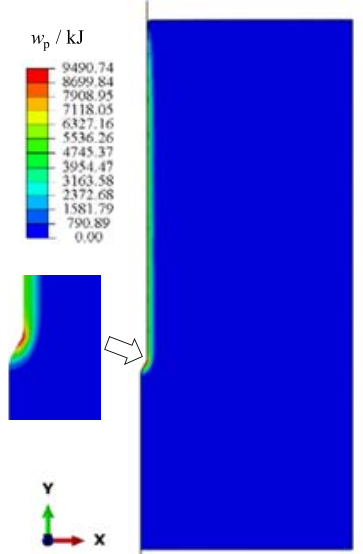

(c)

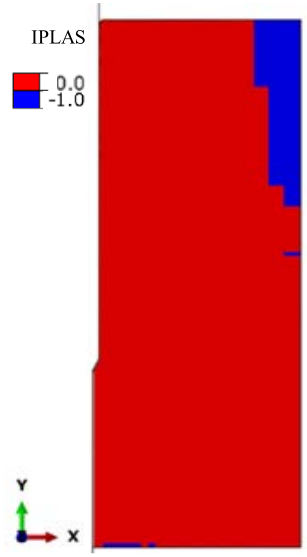

(d)

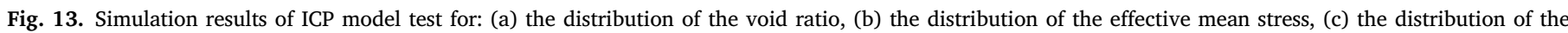
modified plastic work, and (d) the plastic index for activated yielding mechanism.

(a)
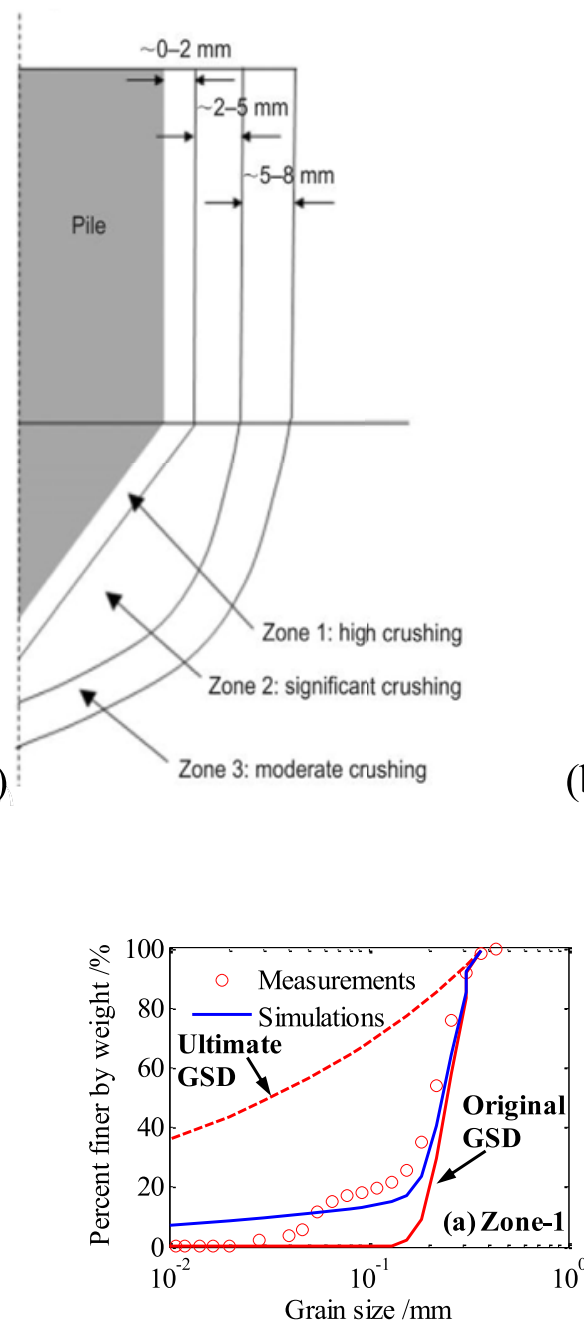

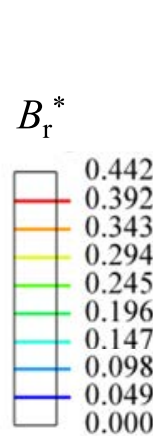

(b)

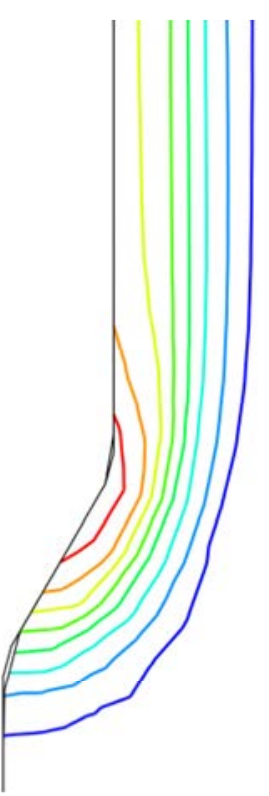

Fig. 14. Comparison between experiment and simulation of model pile test in Fontainebleau sand for the profile of crushing zones: (a) experiment; (b) simulation.

Fig. 15. Comparison between experiment and simulation for the average grain size distributions for (a) Zone 1 , (b) Zone 2 , and (c) Zone 3 .

Fig. 14 shows the particle crushing profiles corresponding to a depth of $1 \mathrm{~m}$ along the model pile. Compared to the observations, the crushing zone simulated by the model is within approximately half a radius of the pile from the shaft, which is in good agreement with the experiments. To evaluate the capability of the adopted breakage model, the grain size distributions of the crushed zones from Zone- 1 to Zone- 3 have been plotted by using Eq. (13) and are presented in Fig. 15, showing also in this case a good agreement with the experimental observations.

Such a result further confirms the ability of the constitutive equations not only to capture the mechanical behavior of sand during the pile 

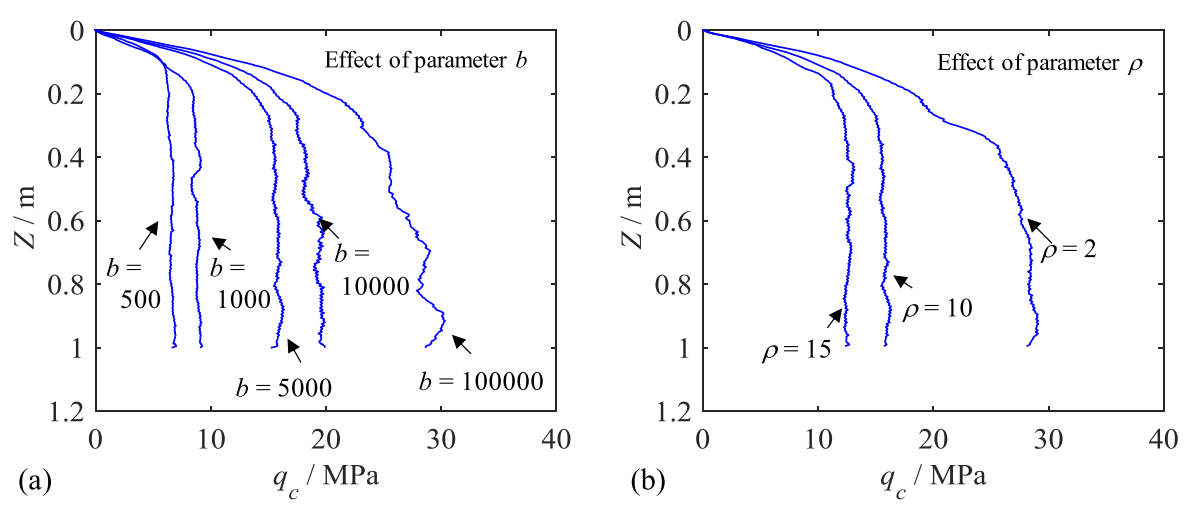

Fig. 16. Influence of breakage related parameters on pile resistance to investigate: (a) the effect of crushability of sand by $b$, (b) the effect of sensitivity of CSL change due to grain breakage, (c) the effect of dilatancy amount by $A_{\mathrm{d}}$, and (d) the effect of dilatancy interlocking by $n_{\mathrm{d}}$.
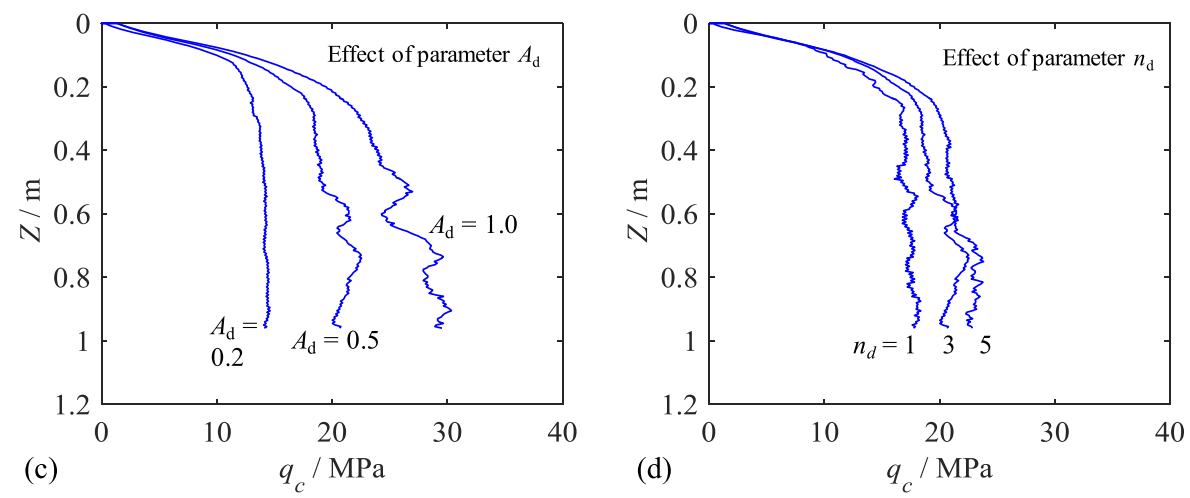

process but also to give an accurate description of the crushed material around the pile, thus improving the understanding of the effect of grain breakage to evaluate pile resistance in engineering design.

To investigate the influence of the breakage on pile resistance, a parametric analysis was further conducted based on the simulation of the ICP model test. For this purpose, two different parameters have been tested: i) the parameter $b$ controlling the evolution rate of the grain breakage index $B_{\mathrm{r}}{ }^{*}$ and ii) the parameter $\rho$ controlling the degradation rate of the CSL due to grain breakage. The simulated pile resistance $q_{\mathrm{c}}$ against the penetration depth with varying value of $b$ has been shown in Fig. 16(a) for different values of the parameters $b$ (i.e., $b=500,1000,5000,50000$, and 100000). From this figure, it is possible to observe that i) the effect of the breakage tends to be negligible for high values of $b$, ii) for small values of $b$ the development of crushing results in a strong decrease of the pile resistance. Similar simulations were also conducted by changing only the parameter $\rho$ (i.e., $\rho=5,10$, and 15) with the related results plotted in Fig. 16(b). It can be seen that pile resistance decreases with an increase in the value of $\rho$ (representing CSL more easily moving down). In both parametric studies performed by changing the parameters $b$ and $\rho$, it is possible to deduce that the effect of grain breakage has a significant influence on the pile resistance. Overall, the effect of grain breakage by changing of parameters $b$ and $\rho$ has a significant influence on the pile resistance.
Furthermore, to highlight the effect of the stress dilatancy during pile penetration, a parametric study on $A_{\mathrm{d}}$ and $n_{\mathrm{d}}$ has also been performed. For this, several simulations were conducted by only changing the parameter $A_{\mathrm{d}}\left(A_{\mathrm{d}}=0.5,1.0\right.$ and 1.5$)$ or only changing the parameter $n_{\mathrm{d}}$ $\left(n_{\mathrm{d}}=1,3\right.$ and 5 ) while keeping other parameters constant. The simulated $q_{c}$ against the penetration depth is shown in Fig. 16(c) and (d) with varying value of $A_{\mathrm{d}}$ and $n_{\mathrm{d}}$, respectively. It can be concluded the increase of both the parameter $A_{\mathrm{d}}$ controlling the dilatancy amount and the parameter $n_{\mathrm{d}}$ controlling the dilatancy interlocking can result in the increase of pile resistance. In common engineering practice, a granular foundation soil with high stress dilatancy gives high pile resistance.

\subsection{Modeling of centrifuge pile penetration}

To further validate the numerical platform, a series of centrifuge pile penetration tests on Fontainebleau NE34 sand with various relative densities $D_{\mathrm{r}}$ (four tests MWG-3, 5, 8 and 9 in Bolton and Gui [56] and five tests CUED, DIA, ISMES, LCPC and RUB in Bolton et al. [57]) were selected and simulated.

Some details of centrifuge pile penetration tests on Fontainebleau NE34 sand at $70 \mathrm{~g}$ are shown in Table 4 . In the simulation, the prototype of pile penetration corresponding to the centrifuge test under $70 \mathrm{~g}$ was

Table 4

A series of centrifuge pile penetrating tests on Fontainebleau sand.

\begin{tabular}{|c|c|c|c|c|c|c|c|c|c|}
\hline Tests (*) & MWG-3 & MWG-5 & MWG-8 & MWG-9 & CUED & DIA & ISMES & LCPC & RUB \\
\hline$B: \mathrm{mm}$ & 10 & 10 & 10 & 10 & 10 & 12 & 11.3 & 12 & 11.3 \\
\hline$D: \mathrm{mm}$ & 850 & 850 & 850 & 850 & 850 & 530 & 400 & 800 & 750 \\
\hline$D / B$ & 85 & 85 & 85 & 85 & 85 & 45 & 35 & 66 & 66 \\
\hline$D_{\mathrm{r}}: \%$ & $81 \%$ & $89 \%$ & $58 \%$ & $54 \%$ & $81 \%$ & $81 \%$ & $84 \%$ & $84 \%$ & $84 \%$ \\
\hline$e_{0}$ & 0.62 & 0.59 & 0.70 & 0.72 & 0.62 & 0.62 & 0.60 & 0.60 & 0.60 \\
\hline
\end{tabular}

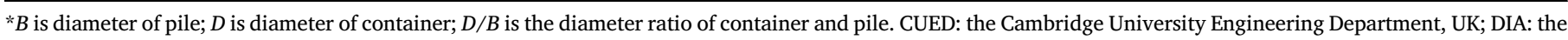

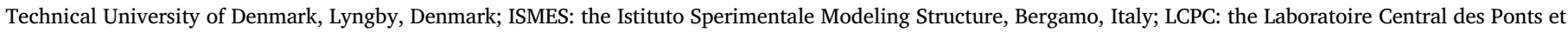
Chausseaes, Nantes, France; RUB: the Ruhr-Universitat, Bochum, Germany. 


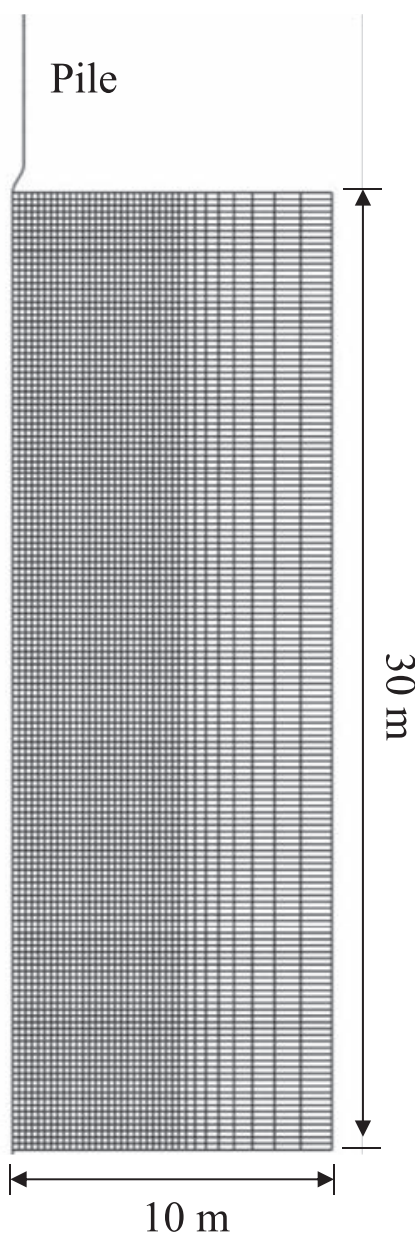

Fig. 17. Geometry of centrifuge pile penetration tests.

adopted. The size of the axisymmetric FE model is $10 \mathrm{~m}$ in radius and $30 \mathrm{~m}$ in depth. The pile has a diameter $B$ of $0.7 \mathrm{~m}$ with an apex angle of $60^{\circ}$. According to the experimental results [57], the boundary effect can be ignored for the FE model shown in Fig. 17 with the diameter ratio $D / B=28.6$. The bottom side of the model is fixed in both vertical and horizontal directions. The left and right sides are fixed only in the horizontal direction. The top side is free. The mesh around the pile is dense enough to allow an accurate result. The initial vertical stress was calculated by the self-weight of Fontainebleau NE34 sand, whereas the horizontal stress was calculated by using $K_{0}=1-\sin \phi^{\prime}=0.48$. The contact type and the friction coefficient were set to be the same as that used in the simulation of the ICP model test. The total penetration displacement is $20 \mathrm{~m}$ with a slow penetration speed of $0.05 \mathrm{~m} / \mathrm{s}$. The parameters for Fontainebleau NE34 sand in Table 3 in the simulations.

Fig. 18 shows the comparisons of pile resistance between experimental results and simulations for the tests MWG-3, 5, 8, and 9 and for the tests CUED, DIA, ISMES, LCPC, and RUB. In this figure, to emphasize the importance of crushing during the penetration process, the numerical results obtained without considering the breakage effects in the model has been also included in the plot. These comparisons indicate also that the grain breakage occurring during the pile penetration can significantly reduce the pile resistance, thus suggesting that the effect of particle fragmentation should be considered during the engineering practice of pile design. Furthermore, the comparisons also indicate that the grain breakage occurring during the pile penetration can significantly reduce the pile resistance, which should be attended to in pile engineering.

Pile resistance $q_{\mathrm{c}}$ was normalized by the effective vertical stress $\sigma_{\mathrm{V}}^{\prime}$ of its penetration depth by Eq. (20), and the penetration depth $Z$ was normalized by the diameter of pile $B$. Then Fig. 18 was replotted in Fig. 19 to draw comparisons of normalized pile resistance among simulations and experiments for all selected centrifuge tests,

$Q=\frac{q_{c}-\sigma_{v}}{\sigma_{v}^{\prime}}$

where $\sigma_{\mathrm{v}}$ and $\sigma_{\mathrm{v}}^{\prime}$ are the total and effective vertical stress, and in this study $\sigma_{\mathrm{v}}^{\prime}=\sigma_{\mathrm{v}}=\gamma \cdot Z$.

It can be seen that the "yield" point of normalized pile resistance $Q$ is obtained around the normalized penetration depth $Z / B$ of 10 for three relative densities of sand, which can be well predicted by the proposed numerical platform. Thus the proposed platform was validated to predict the bearing capacity of pile in practice.

Fig. 20 compares grain size distribution between calculation and measurement-which, again, shows a good agreement for average grain size distribution in the breakage zone.

Overall, all comparisons demonstrate that the proposed numerical platform performs well at simulating pile penetration that involves grain breakage in real practice.

\subsubsection{Correlation of pile resistance with the proposed numerical platform}

To allow investigation of the relationship between relative density and maximum normalized pile resistance, a series of pile penetration tests on Fontainebleau NE34 sand with different relative densities were simulated on the basis of a centrifuge test. Because the sand involved in
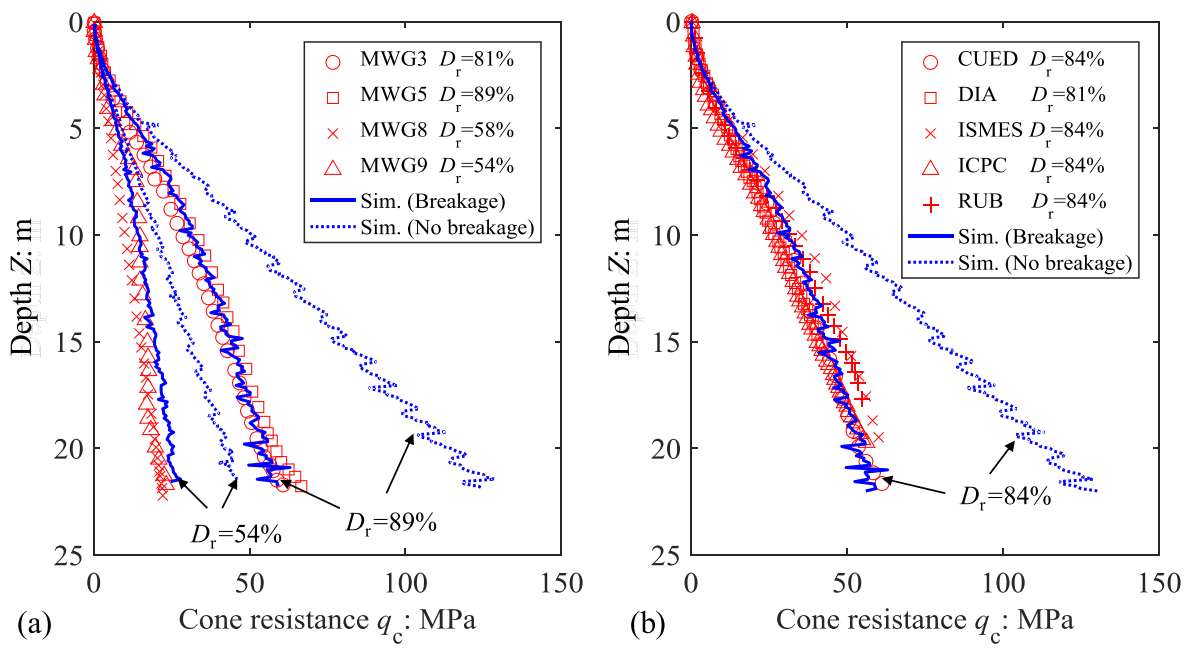

Fig. 18. Comparison between experiments and simulations of centrifuge pile tests in Fontainebleau sand for pile resistance against penetration depth. 

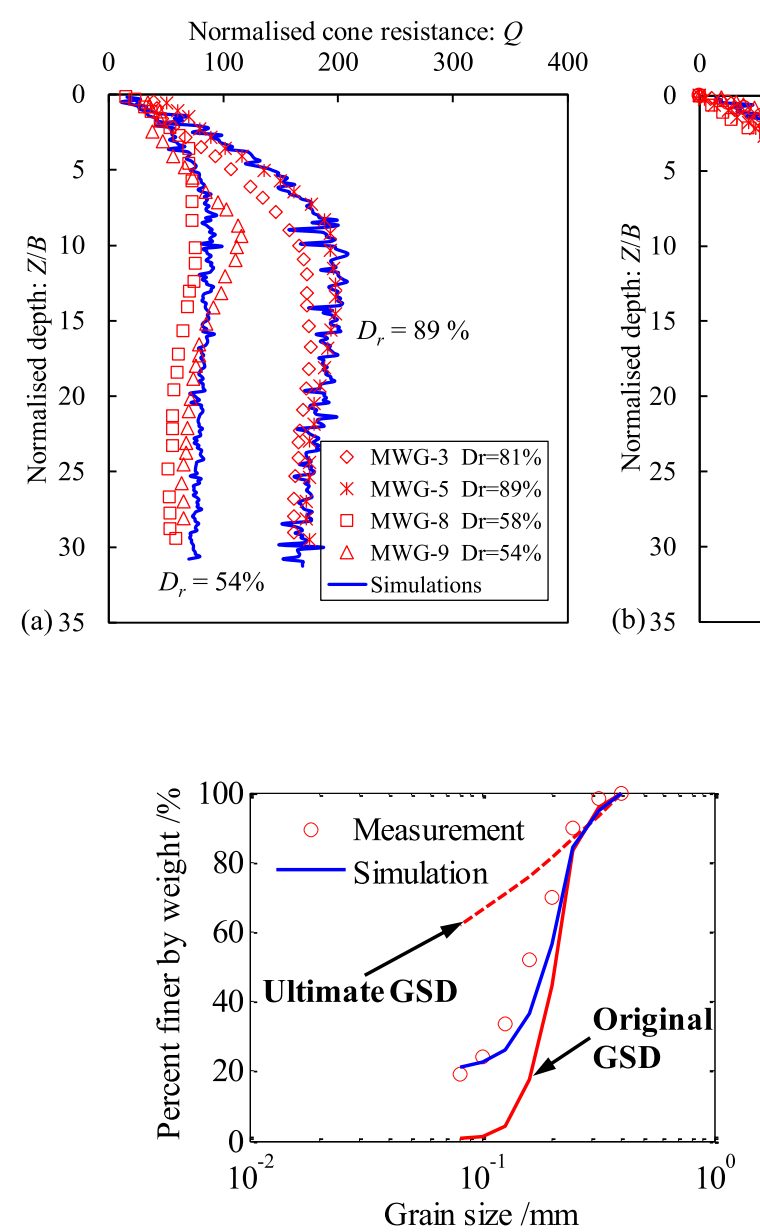

Fig. 20. Comparison of grain size distribution for Fontainebleau sand around pile between simulation and measurement for the case $D_{\mathrm{r}}=84 \%$ (CUED, DIA, ISMES, LCPC and RUB).

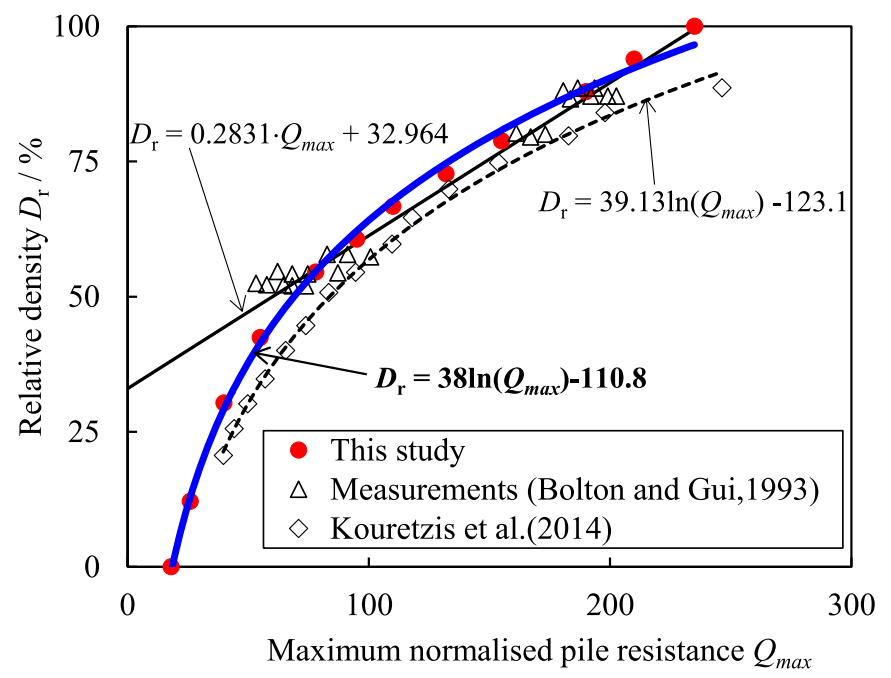

Fig. 21. Relationship between relative density $D_{\mathrm{r}}$ and maximum normalized pile resistance $Q_{\max }$.

the simulation varied from very loose $\left(D_{\mathrm{r}}=0 \%\right)$ to very dense $\left(D_{\mathrm{r}}=100 \%\right)$, additional simulations were carried out that considered grain breakage. Fig. 21 presents the simulated maximum normalized pile resistance $Q_{\max }$ versus the corresponding $D_{\mathrm{r}}$, with good agreement

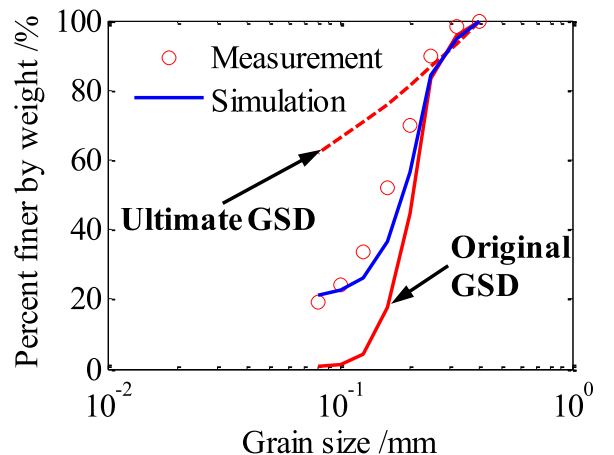

Grain size $/ \mathrm{mm}$
Fig. 19. Comparison between experiments and simulations for normalized pile resistance for centrifuge pile tests.

\section{Conclusions}

An enhanced platform of large deformation analysis that considers the effects of grain breakage during pile penetration in silica sand has been proposed. A multi-surface plastic model accounting for grain breakage was proposed and implemented in the proposed platform. A slight modification of nonlinear CSL was made using the logarithmic scale of void ratio instead of normal scale to guarantee the positiveness of the critical void ratio at a high level of stress. The original cutting plane algorithm for a single-yield surface was extended for models with multiple yielding mechanisms, and the model was finally implemented into the finite element code.

Then, to validate the performance of the proposed platform when simulating pile penetration, a model pile penetration test and a series of centrifuge pile penetration tests on Fontainebleau NE34 sand were selected for simulation. The model parameters of Fontainebleau NE34 sand were calibrated on compression tests and drained triaxial tests, and the simulated results were compared with the experimental measurements to evaluate the performance of the proposed platform. All comparisons demonstrate that not only the laboratory model test of pile penetration but also the centrifuge pile penetration tests could be well predicted. Moreover, the breakage behavior of sand during the pile penetration was also accurately predicted.

Finally, some extra features of the enhanced platform were discussed while conducting the parametric study, such as the effect of sand crushability on pile resistance as measured by parametric analysis of two breakage-related parameters and the pile resistance nonlinearity versus 
sand density. The results demonstrate that (1) the effects on grain breakage of changes to parameters $b$ and $\rho$ significantly influence pile resistance and (2) the relationship between relative density and maximum normalized pile resistance is nonlinear. All comparisons demonstrate that the enhanced platform is applicable to pile foundation design.

\section{Acknowledgement}

The financial support for this research came from the National Natural Science Foundation of China (Grant no. 51579179).

\section{Appendix A. derivatives of constitutive equations}

The derivatives for the numerical integration described in the paper are briefly presented for numerical analyses. The derivative of yield function on the increment of stress vector can be expressed as,

$$
\begin{aligned}
\frac{\partial f}{\partial \sigma_{i j}} & =\frac{\partial f}{\partial p^{\prime}} \frac{\partial p^{\prime}}{\partial \sigma_{i j}}+\frac{\partial f}{\partial q} \frac{\partial q}{\partial \sigma_{i j}} \\
\frac{\partial f}{\partial p^{\prime}} & =-\frac{q}{p^{\prime 2}} \\
\frac{\partial f}{\partial q} & =\frac{1}{p^{\prime}} \\
\frac{\partial p^{\prime}}{\partial \sigma_{i j}} & =\left[\begin{array}{llllll}
\frac{1}{3} & \frac{1}{3} & \frac{1}{3} & 0 & 0 & 0
\end{array}\right]^{T} \\
\frac{\partial q}{\partial \sigma_{i j}} & =\frac{3 s_{i j}}{2 q}=\frac{3}{2 q}\left[\begin{array}{llllll}
\sigma_{11}-p^{\prime} & \sigma_{22}-p^{\prime} & \sigma_{33}-p^{\prime} & \sigma_{12} & \sigma_{13} & \sigma_{23}
\end{array}\right]^{T}
\end{aligned}
$$

The double index $i j$ is simplified as $1 \hat{=} 11,2 \hat{=} 22,3 \hat{=} 33,4 \widehat{=} 12,5 \hat{=} 23,6 \widehat{=} 31$.

The derivative of yield function on the hardening parameters can be expressed as,

$$
\frac{\partial f}{\partial \kappa^{*}}=\frac{\partial f}{\partial \varepsilon_{d}^{p l}}=\frac{-k_{p} M_{p}}{\left(k_{p}+\varepsilon_{d}^{p l}\right)^{2}}
$$

The derivative of hardening parameters on plastic strain can be expressed as,

$$
\frac{\partial \kappa^{*}}{\partial \varepsilon^{p l}}=\frac{\partial \varepsilon_{d}^{p l}}{\partial \varepsilon_{d}^{p l}}=1
$$

The derivative of potential function on increment of stress vector can be expressed as,

$$
\begin{aligned}
& \frac{\partial g}{\partial \sigma_{i j}}=\frac{\partial g}{\partial p^{\prime}} \frac{\partial p^{\prime}}{\partial \sigma_{i j}}+\frac{\partial g}{\partial q} \frac{\partial q}{\partial \sigma_{i j}} \\
& \frac{\partial g}{\partial p^{\prime}}=A_{d}\left(M_{p t}-\frac{q}{p^{\prime}}\right) \\
& \frac{\partial g}{\partial q}=-1
\end{aligned}
$$

\section{Appendix B. Optimization-based parameter identification procedure}

\section{Error function}

The average difference between the measured and the simulated results is expressed in the form of the least square method,

$$
\operatorname{Error}(x)=\sqrt{\frac{1}{N} \sum_{i=1}^{N} w_{i}\left(\frac{U_{\exp }^{i}-U_{n u m}^{i}}{U_{\exp }^{i}}\right)^{2}} \times 100
$$

where $N$ is the number of values; $U_{\exp }^{i}$ is the value of the measurement point $i$; $U_{\text {num }}^{i}$ is the value of the calculation at point $i$; $w_{i}$ is weight for the calculation at point $i$.

Procedure

Fig. B1 shows the procedure of optimization-based parameter identification. 


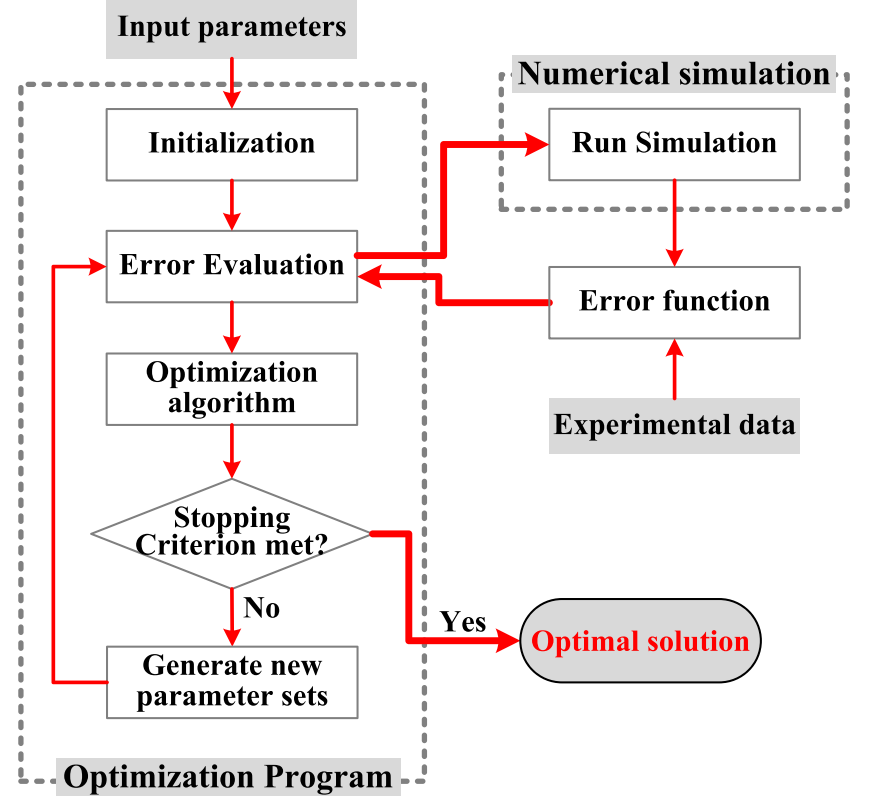

Fig. B1. Procedure of optimization-based parameter identification.

\section{References}

[1] G.P. Kouretzis, D. Sheng, D. Wang, Numerical simulation of cone penetration testing using a new critical state constitutive model for sand, Comput. Geotech. 56 (2014) 50-60.

[2] D. Sheng, K.D. Eigenbrod, P. Wriggers, Finite element analysis of pile installation using large-slip frictional contact, Comput. Geotech. 32 (2005) 17-26.

[3] C. Zhang, G. Nguyen, I. Einav, The end-bearing capacity of piles penetrating into crushable soils, Geotechnique 63 (2013) 341-354.

[4] C. Zhang, Z. Yang, G. Nguyen, R. Jardine, I. Einav, Theoretical breakage mechanics and experimental assessment of stresses surrounding piles penetrating into dense silica sand, Géotech. Lett. 4 (2014) 11-16.

[5] S.-L. Shen, Y.-S. Xu, Numerical evaluation of land subsidence induced by groundwater pumping in Shanghai, Can. Geotech. J. 48 (2011) 1378-1392.

[6] Y.-X. Wu, S.-L. Shen, D.-J. Yuan, Characteristics of dewatering induced drawdown curve under blocking effect of retaining wall in aquifer, J. Hydrol. 539 (2016) $554-566$.

[7] Z. Jin, Z.-Y. Yin, P. Kotronis, Y.-F. Jin, Numerical analysis of a suction bucket penetrating in sand with a combined Lagrangian-SPH approach, Proc. Eng. 175 (2017) 189-196.

[8] S. Lobo-Guerrero, L.E. Vallejo, Influence of pile shape and pile interaction on the crushable behavior of granular materials around driven piles: DEM analyses, Granul. Matter 9 (2007) 241-250.

[9] L. Vallejo, S. Lobo-Guerrero, DEM analysis of crushing around driven piles in granular materials, Geotechnique (2005) 617-623.

[10] S.-L. Shen, Z.-F. Wang, J. Yang, C.-E. Ho, Generalized approach for prediction of jet grout column diameter, J. Geotech. Geoenviron. Eng. 139 (2013) 2060-2069.

[11] S. Shen, Z. Wang, W. Cheng, Estimation of lateral displacement induced by jet grouting in clayey soils, Geotechnique 67 (2017) 621-630.

[12] K. Kuwajima, M. Hyodo, A.F. Hyde, Pile bearing capacity factors and soil crushabiity, J. Geotech. Geoenviron. Eng. 135 (2009) 901-913.

[13] H.-S. Yu, Cavity Expansion Methods in Geomechanics, Springer Science \& Business Media, 2013.

[14] N. Yamamoto, M.F. Randolph, I. Einav, Numerical study of the effect of foundation size for a wide range of sands, J. Geotech. Geoenviron. Eng. 135 (2009) 37-45.

[15] H. Poulos, F. Aust, E. Chua, Bearing Capacity on Calcareous Sand, Research Report University of Sydney, 1986.

[16] R.J. Jardine, B. Zhu, P. Foray, C.P. Dalton, Experimental arrangements for investigation of soil stresses developed around a displacement pile, Soils Found. 49 (2009) 661-673.

[17] Z. Yang, R. Jardine, B. Zhu, P. Foray, C. Tsuha, Sand grain crushing and interface shearing during displacement pile installation in sand, Geotechnique 60 (2010) $469-482$.

[18] C. Zhang, I. Einav, G.D. Nguyen, A study of grain crushing around penetrating piles using a micromechanics-based continuum model, in: Computational Geomechanics, COMGEO II-proceedings of the 2nd International Symposium on Computational Geomechanics, 2011, pp. 714-720.
[19] C. Zhang, Z. Yang, G. Nguyen, R. Jardine, I. Einav, Theoretical breakage mechanics and experimental assessment of stresses surrounding piles penetrating into dense silica sand, Géotech. Lett. 4 (2014) 11-16.

[20] J. Alba, J. Audibert, Pile design in calcareous and carbonaceous granular materials, and historic review, in: Proceedings of the 2nd International Conference on Engineering for Calcareous Sediments Rotterdam: AA Balkema, 1999, pp. 29-44.

[21] J.D. Murff, Pile capacity in calcareous sands: state if the art, J. Geotech. Eng. 113 (1987) 490-507.

[22] E. Susila, R.D. Hryciw, Large Displacement FEM Modelling of the Cone Penetration Test (CPT) in Normally Consolidated Sand, 2003.

[23] T. Hamann, G. Qiu, J. Grabe, Application of a Coupled Eulerian-Lagrangian approach on pile installation problems under partially drained conditions, Comput. Geotech. 63 (2015) 279-290.

[24] G. Qiu, S. Henke, J. Grabe, Application of a Coupled Eulerian-Lagrangian approach on geomechanical problems involving large deformations, Comput. Geotech. 38 (2011) 30-39.

[25] W. Hu, Z.Y. Yin, C. Dano, P.Y. Hicher, A constitutive model for granular materials considering grain breakage, Sci. China Technol. Sci. 54 (2011) 2188-2196.

[26] Z.-Y. Yin, Q. Xu, P.-Y. Hicher, A simple critical-state-based double-yield-surface model for clay behavior under complex loading, Acta Geotech. 8 (2013) 509-523.

[27] F.E. Richart, J.R. Hall, R.D. Woods, Vibrations of Soils and Foundations, 1970.

[28] X.S. Li, Y. Wang, Linear representation of steady-state line for sand, J. Geotech. Geoenviron. Eng. 124 (1998) 1215-1217.

[29] D. Sheng, S. Sloan, H. Yu, Aspects of finite element implementation of critical state models, Comput. Mech. 26 (2000) 185-196.

[30] Y.-P. Yao, N.-D. Wang, Transformed stress method for generalizing soil constitutive models, J. Eng. Mech. 140 (2013) 614-629.

[31] Y. Yao, W. Hou, A. Zhou, UH model: three-dimensional unified hardening model for overconsolidated clays, Geotechnique 59 (2009) 451-469.

[32] Y. Yao, D. Lu, A. Zhou, B. Zou, Generalized non-linear strength theory and transformed stress space, Sci. China, Ser. A: Technol. Sci. 47 (2004) 691-709.

[33] Y. Yao, D. Sun, T. Luo, A critical state model for sands dependent on stress and density, Int. J. Numer. Anal. Meth. GeoMech. 28 (2004) 323-337.

[34] Y.-J. Liu, G. Li, Z.-Y. Yin, C. Dano, P.-Y. Hicher, X.-H. Xia, J.-H. Wang, Influence of grading on the undrained behavior of granular materials, CR Mec 342 (2014) 85-95.

[35] M. Coop, K. Sorensen, T.B. Freitas, G. Georgoutsos, Particle breakage during shearing of a carbonate sand, Geotechnique 54 (2004) 157-163.

[36] A. Daouadji, P.Y. Hicher, An enhanced constitutive model for crushable granular materials, Int. J. Numer. Anal. Meth. GeoMech. 34 (2010) 555-580.

[37] A. Daouadji, P.Y. Hicher, A. Rahma, Modelling grain breakage influence on mechanical behaviour of granular media, Eur. J. Mech. Solid. 20 (2001) 113-137.

[38] W. Hu, Z. Yin, C. Dano, P.-Y. Hicher, A constitutive model for granular materials considering grain breakage, Sci. China Technol. Sci. 54 (2011) 2188-2196.

[39] Karlsson Hibbitt, Sorensen, ABAQUS/Explicit: User's Manual: Hibbitt, Karlsson and Sorenson, 2001.

[40] M. Ortiz, J. Simo, An analysis of a new class of integration algorithms for elastoplastic constitutive relations, Int. J. Numer. Meth. Eng. 23 (1986) 353-366.

[41] C. Gaudin, F. Schnaid, J. Garnier, Sand characterization by combined centrifuge and laboratory tests, Int. J. Phys. Model. Geotech. 5 (2005) 42-56. 
[42] Z.-X. Wu, Z.-Y. Yin, Y.-F. Jin, X.-Y. Geng, A straightforward procedure of parameters determination for sand: a bridge from critical state based constitutive modelling to finite element analysis, Eur. J. Environ. Civil Eng. (2017) 1-23.

[43] Y.-F. Jin, Z.-X. Wu, Z.-Y. Yin, J.S. Shen, Estimation of critical state-related formula in advanced constitutive modeling of granular material, Acta Geotech. 12 (2017) 1329-1351.

[44] Y.-F. Jin, Z.-Y. Yin, S.-L. Shen, P.-Y. Hicher, Investigation into MOGA for identifying parameters of a critical-state-based sand model and parameters correlation by factor analysis, Acta Geotech. 11 (2016) 1131-1145.

[45] Y.-F. Jin, Z.-Y. Yin, S.-L. Shen, P.-Y. Hicher, Selection of sand models and identification of parameters using an enhanced genetic algorithm, Int. J. Numer. Anal. Meth. GeoMech. 40 (2016) 1219-1240.

[46] Y.-F. Jin, Z.-Y. Yin, S.-L. Shen, D.-M. Zhang, A new hybrid real-coded genetic algorithm and its application to parameters identification of soils, Inverse Probl. Sci. Eng. 25 (2017) 1343-1366.

[47] Z.-Y. Yin, Y.-F. Jin, J.S. Shen, P.-Y. Hicher, Optimization techniques for identifying soil parameters in geotechnical engineering: comparative study and enhancement, Int. J. Numer. Anal. Meth. GeoMech. 42 (2018) 70-94.

[48] Z.-Y. Yin, Y.-F. Jin, S.-L. Shen, H.-W. Huang, An efficient optimization method fo identifying parameters of soft structured clay by an enhanced genetic algorithm and elastic-viscoplastic model, Acta Geotech. 12 (2017) 849-867.
[49] A. Touati, Comportement mécanique des sols pulvérulents sous fortes contraintes, These presentee a l'ecole nationale des ponts et chaussees pour obtenir le diplome de docteur-ingenieur, 1982.

[50] Z.-Y. Yin, P.-Y. Hicher, C. Dano, Y.-F. Jin, Modeling mechanical behavior of very coarse granular materials, J. Eng. Mech. 143 (2017). C4016006.

[51] P.A. Bopp, P.V. Lade, Effects of initial density on soil instability at high pressures, J. Geotech. Geoenviron. Eng. 123 (1997) 671-677.

[52] P.V. Lade, P.A. Bopp, Relative density effects on drained sand behavior at high pressures, Soils Found. 45 (2005) 1-13.

[53] P.V. Lade, J.A. Yamamuro, P.A. Bopp, Significance of particle crushing in granular materials, J. Geotech. Eng. 122 (1996) 309-316.

[54] A.R. Russell, N. Khalili, A bounding surface plasticity model for sands exhibiting particle crushing, Can. Geotech. J. 41 (2004) 1179-1192.

[55] J.A. Yamamuro, P.V. Lade, Drained sand behavior in axisymmetric tests at high pressures, J. Geotech. Eng. 122 (1996) 109-119.

[56] M. Bolton, M. Gui, The Study of Relative Density and Boundary Effects for Cone Penetration Tests in Centrifuge, University of Cambridge, Department of Engineering, 1993.

[57] M.D. Bolton, M.-W. Gui, J. Garnier, J.F. Corte, G. Bagge, J. Laue, R. Renzi, Centrifuge cone penetration tests in sand, Geotechnique 49 (1999) 543-552. 